\title{
Description of the two neutrino double $\beta$ decay in deformed nuclei with projected spherical single particle basis
}

\author{
A. A. Raduta, ${ }^{\mathrm{a}, \mathrm{b}}$ C. M. Raduta, ${ }^{\mathrm{b}}$ and A. Escuderos ${ }^{\mathrm{c}, \mathrm{d}}$ \\ a Department of Theoretical Physics and Mathematics, Bucharest University, P.O. Box MG11, Romania \\ ${ }^{\mathrm{b}}$ Institute of Physics and Nuclear Engineering, Bucharest, P.O. Box MG6, Romania \\ ${ }^{\mathrm{c}}$ Instituto de Estructura de la Materia, Consejo Superior de Investigationes Cientificas, Serrano 123, E-28006 Madrid, Spain \\ ${ }^{\mathrm{d}}$ Department of Physics and Astronomy, Rutgers University, Piscataway, New Jersey 00854, USA
}

(Received 27 August 2004; published 16 February 2005)

\begin{abstract}
Using an angular-momentum-projected single-particle basis, a proton-neutron quasiparticle random-phase approximation approach is used to study the $2 \nu \beta \beta$ properties of 10 isotopes, exhibiting various quadrupole deformations. The parent and daughter nuclei exhibit different quadrupole deformations. Since the projected basis enables a unified description of deformed and spherical nuclei, situations where the nuclei involved in the double $\beta$ decay process are both spherical, both deformed, or one spherical and the other deformed can be treated through a single formalism. Dependence of single $\beta^{-}$and $\beta^{+}$strength distribution on atomic mass number and nuclear deformation is analyzed. For the double $\beta$ decay process, the Gamow-Teller transition amplitudes and half-lives are calculated. Results are compared with experimental data as well as with predictions of other theoretical approaches. The agreement between the present results and experimental data is fairly good.
\end{abstract}

DOI: 10.1103/PhysRevC.71.024307

PACS number(s): $23.40 . \mathrm{Hc}$

\section{INTRODUCTION}

The double $\beta$ decay of a $(Z, N)$ nucleus may take place through two distinct modes. In one mode, the final state consists of the residual nucleus $(Z+2, N-2)$, two electrons, and two antineutrinos; in the other mode the final state lacks the antineutrinos. Appropriately, the two decay modes are called two neutrino double $\beta(2 v \beta \beta)$ and neutrinoless $(0 v \beta \beta)$ double $\beta$ decay, respectively. The second mode is especially interesting since its discovery may provide a definite answer to the question of whether the neutrino is a Dirac or Majorana particle. The $2 \nu \beta \beta$ process is interesting to study not only for its own merits but also because it may provide realistic nuclear matrix elements that might be further used to quantitatively evaluate the rate of the neutrinoless double $\beta$ decay.

For such reasons many theoreticians have focused their efforts on describing consistently the data for $2 \nu \beta \beta$ decay. The contributions over several decades have been reviewed by many authors. Therefore, instead of enumerating the main steps achieved toward improving the theoretical description, we advise the reader to consult a few of the review works [1-8].

It is interesting to note that although most of the double $\beta$ emitters are deformed nuclei, the proposed formalisms use a single-particle spherical basis. More than 10 years ago, one of us [9] proposed a formalism to describe the process of two neutrino double $\beta$ decay in a projected spherical basis. It was the first time that a proton-neutron quasiparticle random-phase approximation (pnQRPA) for a two-body interaction in the $p h$ and $p p$ channels with a deformed single-particle basis was performed. Moreover, effects that are beyond pnQRPA have been accounted for by means of a boson expansion procedure. A few years later, researchers [10] studied the influence of nuclear deformation on the contribution of the spin-flip configurations to the Gamow-Teller (GT) double $\beta$ transition amplitude. In the meantime, several papers have been devoted to the extension of the pnQRPA procedure to deformed nuclei, the applications being performed to study the single $\beta$ decay properties as well as the double $\beta$ decay rates. Thus, pnQRPA approaches using as the deformed single-particle basis the Nilsson or deformed Woods-Saxon states have been formulated [11-14]. Also, a self-consistent deformed method was formulated where the single-particle basis was obtained as eigenstates of a deformed mean field defined through a Hartree-Fock treatment of a density-dependent two-body interaction of Skyrme type [12].

In a recent publication [15] we continued the project opened in Ref. [9] by improving the single-particle basis. Indeed, in Ref. [9] the single-particle energies depended linearly on a parameter that simulated the nuclear deformation. In contrast, Ref. [15] applied the core volume conservation constraint, ignored in the previous paper, and determined a nonlinear deformation dependence for single-particle energies. Of course, having different single-particle energies, the pairing properties and the double $\beta$ matrix elements are expected to be modified. Another issue addressed in the previous paper was whether considering different deformations for the parent and daughter nuclei modified significantly the double $\beta$ transition amplitude $\left(M_{\mathrm{GT}}\right)$. The answer to this question is positive because by modifying the deformation for the daughter nucleus, the ground-state correlations are modified and consequently the pnQRPA collapse point is changed. On the other hand, the overlap matrix elements of the states describing the intermediate odd-odd nucleus, defined as excited states from the parent and daughter ground states, are decreased. Therefore, considering different nuclear deformations for parent and daughter nuclei quenches the GT double $\beta$ decay amplitude, thereby improving the agreement with experimental data.

The angular-momentum-projected spherical basis enables us to obtain a unified description of spherical and deformed nuclei. Here we use this virtue of the single-particle basis 
defined in Ref. [15] and try to depict the specific features of the transitions between nuclei of similar or different nuclear shapes: spherical to spherical $\left({ }^{48} \mathrm{Ca} \rightarrow{ }^{48} \mathrm{Ti}\right)$, spherical to deformed prolate $\left({ }^{128} \mathrm{Te} \rightarrow{ }^{128} \mathrm{Xe},{ }^{130} \mathrm{Te} \rightarrow{ }^{130} \mathrm{Xe}\right)$, spherical to deformed oblate $\left({ }^{134} \mathrm{Xe} \rightarrow{ }^{134} \mathrm{Ba},{ }^{136} \mathrm{Xe} \rightarrow{ }^{136} \mathrm{Ba}\right)$, deformed to spherical $\left({ }^{110} \mathrm{Pd} \rightarrow{ }^{110} \mathrm{Cd}\right)$, deformed prolate to deformed prolate $\left({ }^{96} \mathrm{Zr} \rightarrow{ }^{96} \mathrm{Mo}\right)$, and deformed oblate to deformed oblate $\left({ }^{100} \mathrm{Mo} \rightarrow{ }^{100} \mathrm{Ru},{ }^{104} \mathrm{Ru} \rightarrow{ }^{104} \mathrm{Pd},{ }^{116} \mathrm{Cd} \rightarrow\right.$ $\left.{ }^{116} \mathrm{Sn}\right)$. It is worth mentioning that experimental data are available for all cases except ${ }^{104} \mathrm{Ru},{ }^{110} \mathrm{Pd}$, and ${ }^{134} \mathrm{Xe}$.

The results of the present study are described according to the following plan. For the sake of a self-sustaining presentation, a brief review of the projected spherical singleparticle basis is presented in Sec. II along with the basic equations necessary for calculating the GT double $\beta$ transition amplitude. In Sec. III, we discuss the results for 10 double $\beta$ emitters, ${ }^{48} \mathrm{Ca},{ }^{96} \mathrm{Zr},{ }^{100} \mathrm{Mo},{ }^{104} \mathrm{Ru},{ }^{110} \mathrm{Pd},{ }^{116} \mathrm{Cd},{ }^{128} \mathrm{Te}$, ${ }^{130} \mathrm{Te},{ }^{134} \mathrm{Xe}$, and ${ }^{136} \mathrm{Xe}$, for which the strength distribution for single $\beta^{-}$emission, the $M_{\mathrm{GT}}$, and half-life values for the double $\beta$ decay process are presented. Also, the strength distributions for the $\beta^{+}$decay of the corresponding daughter nuclei are presented as functions of the pnQRPA energy. A short summary and concluding remarks are given in Sec. IV.

\section{II. pNQRPA TREATMENT OF THE GT $\beta \beta$ TRANSITION AMPLITUDE}

\section{A. Projected single-particle basis}

In Ref. [16], one of us (A.A.R.) introduced an angularmomentum-projected single-particle basis that seems to be appropriate for the description of the single-particle motion in a deformed mean field generated by the particle-core interaction. This single-particle basis has been used to study the collective M1 states in deformed nuclei [17] as well as the rate of the double $\beta$ process $[9,10]$. Recently, a new version has been proposed where the deformation dependence of single-particle energies is nonlinear and therefore more realistic [18,19]. The new single-particle basis has been used to study the double $\beta$ decay of deformed nuclei [15]. To fix the necessary notations and for the sake of completeness, we describe briefly the main ideas underlying the construction of the projected single-particle basis.

The single particle mean field is determined by a particlecore Hamiltonian

$$
\tilde{H}=H_{\text {sm }}+H_{\text {core }}-M \omega_{0}^{2} r^{2} \sum_{\lambda=0,2} \sum_{-\lambda \leqslant \mu \leqslant \lambda} \alpha_{\lambda \mu}^{*} Y_{\lambda \mu},
$$

where $H_{\mathrm{sm}}$ denotes the spherical shell-model Hamiltonian and $H_{\text {core }}$ is a harmonic quadrupole boson $\left(b_{\mu}^{+}\right)$Hamiltonian associated to a phenomenological core. The interaction of the two subsystems is accounted for by the third term of the equation, written in terms of the shape coordinates $\alpha_{00}, \alpha_{2 \mu}$. The quadrupole shape coordinates are related to the quadrupole boson operators by the canonical transformation

$$
\alpha_{2 \mu}=\frac{1}{k \sqrt{2}}\left[b_{2 \mu}^{\dagger}+(-)^{\mu} b_{2,-\mu}\right]
$$

where $k$ is an arbitrary real number. The monopole shape coordinate is to be determined from the volume conservation condition.

Averaging $\tilde{H}$ on a given eigenstate of $H_{\text {sm }}$, denoted as usual by $|n l j m\rangle$, results in a deformed quadrupole boson Hamiltonian whose eigenstate is an axially symmetric coherent state

$$
\Psi_{g}=\exp \left[d\left(b_{20}^{+}-b_{20}\right)\right]|0\rangle_{b},
$$

with $|0\rangle_{b}$ standing for the vacuum state of the boson operators and $d$ a real parameter that simulates the nuclear deformation. On the other hand, by averaging $\tilde{H}$ on $\Psi_{g}$ one obtains a single-particle mean-field operator for the single-particle motion, similar to the Nilsson Hamiltonian. Finally, averaging on a factor state of the particle-core space breaks the rotational symmetry, and the mean field mentioned above may generate, by diagonalization, a deformed basis for treating the manybody interacting systems. However, this standard procedure is tedious because the final many-body states should be projected over angular momentum.

Our procedure first defines a spherical basis for the particlecore system by projecting the angular momentum out from the deformed state

$$
\Psi_{n l j}^{\mathrm{pc}}=|n l j m\rangle \Psi_{g} .
$$

The upper index appearing on the left-hand side of (2.4) suggests that the product function is associated with the particle-core system. The projected states are obtained, in the usual manner, by acting on these deformed states with the projection operator

$$
P_{M K}^{I}=\frac{2 I+1}{8 \pi^{2}} \int D_{M K}^{I}{ }^{*}(\Omega) \hat{R}(\Omega) d \Omega .
$$

We consider the subset of projected states

$$
\Phi_{n l j}^{I M}(d)=\mathcal{N}_{n l j}^{I} P_{M I}^{I}\left[|n l j I\rangle \Psi_{g}\right] \equiv \mathcal{N}_{n l j}^{I} \Psi_{n l j}^{I M}(d),
$$

which are orthonormalized and form a basis for the particlecore system. The main properties of these projected spherical states are as follows:

1. They are orthogonal with respect to $I$ and $M$ quantum numbers.

2. Although the projected states are associated with the particle-core system, they can be used as a singleparticle basis. Indeed, when a matrix element of a particlelike operator is calculated, the integration on the core collective coordinates is performed first, which results in a final factorized expression: one factor carries the dependence on deformation and one is a spherical shellmodel matrix element. Thus, the role of the core component is to induce a quadrupole deformation for the matrix elements of the operators acting on particle degrees of freedom.

3. The connection between the nuclear deformation and the parameter $d$ entering the definition of the coherent state (2.3) can be obtained by requiring that the strength of the particle-core quadrupole-quadrupole interaction be identical to the Nilsson deformed term of the mean field.

To the projected spherical states, one associates the deformed single-particle energies defined as average values of 
the particle-core Hamiltonian $H^{\prime}=\tilde{H}-H_{\text {core }}$, that is,

$$
\epsilon_{n l j}^{I}=\left\langle\Phi_{n l j}^{I M}(d)\left|H^{\prime}\right| \Phi_{n l j}^{I M}(d)\right\rangle .
$$

Since the core contribution to this average value does not depend on the quantum numbers of the single-particle energy levels, it produces a constant shift for all energies. For this reason such a term is omitted in (2.7). However, when the ground-state energy variation against deformation is studied, this term must be included.

Ref. [15] showed that single-particle energies, defined above, exhibit a nonlinear dependence on the deformation parameter $d$. Such a dependence is determined by the monopole-monopole interaction term after implementing the volume conservation constraint. Moreover, the deformation dependence of the new single-particle energies is similar to that shown by the Nilsson model [20]. Therefore, the average values $\epsilon_{n l j}^{I}$ may be viewed as approximate expressions for the single-particle energies in deformed Nilsson orbits [20]. We may account for the deviations from the exact eigenvalues by considering, at a later stage when a specific treatment of the many-body system is performed, the exact matrix elements of the two-body interaction.

Although the energy levels are similar to those of the Nilsson model, the quantum numbers in the two schemes are different. Indeed, here we generate from each $j$ a multiplet of $(2 j+1)$ states distinguished by the quantum number $I$, which plays the role of the Nilsson quantum number $\Omega$ and runs from $1 / 2$ to $j$. Moreover, the energies corresponding to the quantum numbers $K$ and $-K$ are equal to each other. On the other hand, for a given $I$ there are $2 I+1$ degenerate substates, whereas the Nilsson states are only double degenerate. As explained in [16], the redundancy problem can be solved by changing the normalization of the model functions such that

$$
\left\langle\Phi_{\alpha}^{I M} \mid \Phi_{\alpha}^{I M}\right\rangle=1 \Longrightarrow \sum_{M}\left\langle\Phi_{\alpha}^{I M} \mid \Phi_{\alpha}^{I M}\right\rangle=2 .
$$

Due to this weighting factor, the particle density function provides the consistent result that the number of particles which can be distributed on the $(2 I+1)$ substates is at most 2 , which agrees with the Nilsson model. Here $\alpha$ stands for the set of shell-model quantum numbers $n l j$. Because of this normalization, the states $\Phi_{\alpha}^{I M}$ used to calculate the matrix elements of a given operator should be multiplied by the weighting factor $\sqrt{2 /(2 I+1)}$.

In conclusion, the projected single-particle basis is defined by Eq. (2.6). Although these states are associated with a particle-core system, they can be used as a single-particle basis because of the properties listed earlier. The projected states might be thought of as eigenstates of an effective rotational invariant fermionic one-body Hamiltonian $H_{\text {eff }}$, with the corresponding energies given by Eq. (2.7), such that

$$
H_{\mathrm{eff}} \Phi_{\alpha}^{I M}=\epsilon_{\alpha}^{I}(d) \Phi_{\alpha}^{I M} .
$$

This definition should be supplemented by the request that the matrix elements of any operator between states $\Phi_{\alpha}^{I M}$ and $\Phi_{\alpha^{\prime}}^{I^{\prime} M^{\prime}}$ have, as we mentioned above, a factorized form, with one factor carrying the $d$ dependence and the second one being a spherical shell-model matrix element. Because of these features, these states can be used as a singleparticle basis to treat many-body Hamiltonians that involve one-body operators. This is the case of Hamiltonians with two-body separable forces. As a matter of fact, such a type of Hamiltonian is used in the present paper.

As shown in Ref. [15], in the vibrational limit $d \rightarrow 0$, the projected spherical basis goes to the spherical shell-model basis and $\epsilon_{n l j}^{I}$ to the eigenvalues of $H_{\mathrm{sm}}$.

A fundamental result obtained in Ref. [19] for the product of two single-particle states, which comprises a product of two core components, deserves to be mentioned. Therein we proved that the matrix elements of a two-body interaction corresponding to the present scheme were very close to the matrix elements corresponding to spherical states projected from a deformed product state with one factor being a product of two spherical single-particle states, and a second factor consisting of a common collective core wave function. The small discrepancies of the two types of matrix elements could be washed out by using slightly different strengths for the two-body interaction in the two methods. Because of this property, the basis (2.6) might be used for studying any two-body interaction.

\section{B. The model Hamiltonian and its pnQRPA approach}

In the present work, we aim to describe the Gamow-Teller two neutrino double $\beta$ decay processes with the property that parent and daughter nuclei may exhibit different shapes. Indeed, in the chosen cases they might both be spherical or both be deformed but with different deformations, or one may be spherical and the other deformed. The specific feature of the formalism used consists of treating all cases in an unified manner by using a sole single-particle basis.

The main ingredients of our formalism are as follows. The Fermi transitions contribute about $20 \%$ and the "forbidden" transitions are ignored, which is a reasonable approximation for the two neutrino double $\beta$ decay in medium and heavy nuclei. As usual, the $2 \nu \beta \beta$ process is conceived as two successive single $\beta^{-}$transitions. The first transition connects the ground state of the parent nucleus to a magnetic dipole state $1^{+}$of the intermediate odd-odd nucleus which subsequently decays to the ground state of the daughter nucleus. The states mentioned above, involved in the $2 \nu \beta \beta$ process, are described in the framework of the pnQRPA formalism by using the following many-body Hamiltonian:

$$
\begin{aligned}
H= & \sum \frac{2}{2 I+1}\left(\epsilon_{\tau \alpha I}-\lambda_{\tau \alpha}\right) c_{\tau \alpha I M}^{\dagger} c_{\tau \alpha I M}-\sum \frac{G_{\tau}}{4} P_{\tau \alpha I}^{\dagger} P_{\tau \alpha I^{\prime}} \\
& +2 \chi \sum \beta_{\mu}^{-}(p n) \beta_{-\mu}^{+}\left(p^{\prime} n^{\prime}\right)(-)^{\mu} \\
& -2 \chi_{1} \sum P_{1 \mu}^{-}(p n) P_{-\mu}^{+}\left(p^{\prime} n^{\prime}\right)(-)^{\mu} .
\end{aligned}
$$

The operator $c_{\tau \alpha I M}^{\dagger}\left(c_{\tau \alpha I M}\right)$ creates (annihilates) a particle of type $(\tau=p, n)$ in the state $\Phi_{\alpha}^{I M}$, when acting on the vacuum state $|0\rangle$. To simplify the notation, hereafter the set of quantum numbers $\alpha(=n l j)$ will be omitted. The two-body interaction consists of three terms: the pairing, the dipole-dipole particlehole $(p h)$, and the particle-particle $(p p)$ interactions. The corresponding strengths are denoted by $G_{\tau}(\tau=p, n), \chi$, and $\chi_{1}$, 
respectively. All of them are separable interactions, with the factors defined by the following expressions:

$$
\begin{aligned}
P_{\tau I}^{\dagger} & =\sum_{M} \frac{2}{2 I+1} c_{\tau I M}^{\dagger} c_{\tau \widetilde{I M}}^{\dagger}, \\
\beta_{\mu}^{-}(p n) & =\sum_{M, M^{\prime}} \frac{\sqrt{2}}{\hat{I}}\left\langle p I M\left|\sigma_{\mu}\right| n I^{\prime} M^{\prime}\right\rangle \frac{\sqrt{2}}{\hat{I}^{\prime}} c_{p I M}^{\dagger} c_{n I^{\prime} M^{\prime}}, \\
P_{1 \mu}^{-}(p n) & =\sum_{M, M^{\prime}} \frac{\sqrt{2}}{\hat{I}}\left\langle p I M\left|\sigma_{\mu}\right| n I^{\prime} M^{\prime}\right\rangle \frac{\sqrt{2}}{\hat{I}^{\prime}} c_{p I M}^{\dagger} c_{n \widetilde{I}^{\prime} M^{\prime}}^{\dagger} .
\end{aligned}
$$

The remaining operators from Eq. (2.10) can be obtained from these operators by Hermitian conjugation.

The one-body term and the pairing interaction terms are treated first through the standard BCS formalism and consequently replaced by the quasiparticle one-body term $\sum_{\tau I M} E_{\tau} a_{\tau I M}^{\dagger} a_{\tau I M}$. In terms of quasiparticle creation $\left(a_{\tau I M}^{\dagger}\right)$ and annihilation $\left(a_{\tau I M}\right)$ operators, related to the particle operators by means of the Bogoliubov-Valatin transformation, the two-body interaction terms involved in the model Hamiltonian can be expressed just by replacing the operators (2.11) by their quasiparticle images, which in turn can be expressed as the linear combination of dipole two quasiparticle and quasiparticle density operators defined as

$$
\begin{aligned}
A_{1 \mu}^{\dagger}(p n) & =\sum_{m_{p}, m_{n}} C_{m_{p} m_{n} \mu}^{I_{p} I_{n} 1} a_{p I_{p} m_{p}}^{\dagger} a_{n I_{n} m_{n}}^{\dagger}, \\
B_{1 \mu}^{\dagger}(p n) & =\sum_{m_{p}, m_{n}} C_{m_{p}-m_{n} \mu}^{I_{p} I_{n} 1} a_{p I_{p} m_{p}}^{\dagger} a_{n I_{n} m_{n}}(-)^{I_{n}-m_{n}} \\
& =-\left[a_{p I_{p}}^{\dagger} a_{\widetilde{n I} I_{n}}\right]_{1 \mu} .
\end{aligned}
$$

The quasiparticle Hamiltonian is further treated within the pnQRPA formalism; i.e., one determines the operator

$$
\Gamma_{1 \mu}^{\dagger}=\sum_{k}\left[X(k) A_{1 \mu}^{\dagger}(k)-Y(k) A_{1,-\mu}(k)(-)^{1-\mu}\right],
$$

which satisfies the restrictions

$$
\left[\Gamma_{1 \mu}, \Gamma_{1 \mu^{\prime}}^{\dagger}\right]=\delta_{\mu, \mu^{\prime}}, \quad\left[H_{q p}, \Gamma_{1 \mu}^{\dagger}\right]=\omega \Gamma_{1 \mu}^{\dagger} .
$$

These operator equations yield a set of algebraic equations for the $X$ (usually called forward going) and $Y$ (back going) amplitudes:

$$
\begin{gathered}
\left(\begin{array}{cc}
\mathcal{A} & \mathcal{B} \\
-\mathcal{B} & -\mathcal{A}
\end{array}\right)\left(\begin{array}{c}
X \\
Y
\end{array}\right)=\omega\left(\begin{array}{c}
X \\
Y
\end{array}\right), \\
\sum_{k}\left[|X(k)|^{2}-|Y(k)|^{2}\right]=1 .
\end{gathered}
$$

The analytical expressions for the pnQRPA matrices $\mathcal{A}$ and $\mathcal{B}$ are given in Ref. [15]. Since the $p p$ interaction has an attractive character, for a critical value of $\chi_{1}$ the lowest root of the pnQRPA equations may become imaginary. Suppose that $\chi_{1}$ is smaller than its critical value and therefore all RPA solutions (i.e., $\omega$ ) are real numbers and ordered as

$$
\omega_{1} \leqslant \omega_{2} \leqslant \cdots \leqslant \omega_{N_{s}} .
$$

Here $N_{s}$ stands for the total number of the proton-neutron pair states whose angular momenta can couple to $1^{+}$and, moreover, their quantum numbers $n, l$ are the same. Hereafter the phonon amplitudes $X$ and $Y$ will be accompanied by a lower index $i$, suggesting that they correspond to the energy $\omega_{i}$.

Since our single-particle basis states depend on the deformation parameter $d$, so do the pnQRPA energies and amplitudes. The pnQRPA ground state (the vacuum state of the pnQRPA phonon operator) describes an even-even system which might be either the parent or the daughter nucleus. In the two cases the gauge and nuclear deformation properties are different, which results in determining distinct pnQRPA phonon operators acting on different vacua describing the parent and daughter ground states. Therefore, one needs an additional index distinguishing the phonon operators of the parent and daughter nuclei. The single phonon states are defined by the equations

$$
\left|1_{k} \mu\right\rangle_{j}=\Gamma_{j k ; 1 \mu}^{\dagger}|0\rangle_{j}, \quad j=i, f ; k=1,2, \ldots N_{s} .
$$

Here the indices $i$ and $f$ stand for initial (parent) and final (daughter) nuclei, respectively. This equation defines two sets of nonorthogonal states, $\left\{\left|1_{k} \mu\right\rangle_{i}\right\}$, and $\left\{\left|1_{k} \mu\right\rangle_{f}\right\}$, describing the neighboring odd-odd nucleus. The states of the first set may be fed by a $\beta$-minus decay of the ground state of the parent nucleus while the states of the second set are populated with a $\beta$-plus transition operator from the ground state of the daughter nucleus.

If the energy carried by leptons in the intermediate state is approximated by the sum of the rest energy of the emitted electron and half the $Q$ value of the double $\beta$ decay process

$$
\Delta E=\frac{1}{2} Q_{\beta \beta}+m_{e} c^{2},
$$

the reciprocal value of the $2 v \beta \beta$ half-life can be factorized as

$$
\left(T_{1 / 2}^{2 \nu \beta \beta}\right)^{-1}=F\left|M_{\mathrm{GT}}\left(0_{i}^{+} \rightarrow 0_{f}^{+}\right)\right|^{2},
$$

where $F$ is an integral on the phase space, independent of the nuclear structure, and $M_{\mathrm{GT}}$ stands for the Gamow-Teller transition amplitude and has the expression

$$
M_{\mathrm{GT}}=\sqrt{3} \sum_{k k^{\prime}} \frac{{ }_{i}\left\langle 0 \| \beta_{i}^{+}|| 1_{k}\right\rangle_{i i}\left\langle 1_{k} \mid 1_{k^{\prime}}\right\rangle_{f f}\left\langle 1_{k^{\prime}}\left\|\beta_{f}^{+}\right\| 0\right\rangle_{f}}{E_{k}+\Delta E+E_{1^{+}}} .
$$

In (2.21), the denominator consists of three terms: (1) $\Delta E$, which was already defined, (2) the average value of the $k$ th pnQRPA energy normalized to the particular value corresponding to $k=1$, i.e.,

$$
E_{k}=\frac{1}{2}\left(\omega_{i, k}+\omega_{f, k}\right)-\frac{1}{2}\left(\omega_{i, 1}+\omega_{f, 1}\right),
$$

and (3) the experimental energy for the lowest $1^{+}$state. The indices carried by the transition operators indicate that the operators act in the space spanned by the pnQRPA states associated to the initial $i$ or final $f$ nucleus. The overlap matrix elements of the single phonon states in the parent and daughter nuclei are calculated within the pnQRPA approach and have 
TABLE I. The number of single-particle proton states lying above the $(Z, N)$ core. The single-particle space for neutrons is identical to that for protons. $D_{1}$ and $D_{2}$ are the dimensions of the pnQRPA matrix for parent and daughter nuclei, respectively.

\begin{tabular}{lcccccccccc}
\hline \hline Nucleus & ${ }^{48} \mathrm{Ca}$ & ${ }^{96} \mathrm{Zr}$ & ${ }^{100} \mathrm{Mo}$ & ${ }^{104} \mathrm{Ru}$ & ${ }^{110} \mathrm{Pd}$ & ${ }^{116} \mathrm{Cd}$ & ${ }^{128} \mathrm{Te}$ & ${ }^{130} \mathrm{Te}$ & ${ }^{134} \mathrm{Xe}$ & ${ }^{136} \mathrm{Xe}$ \\
\hline$(Z, N)$ core & $(0,0)$ & $(20,20)$ & $(26,26)$ & $(26,26)$ & $(26,26)$ & $(26,26)$ & $(44,44)$ & $(44,44)$ & $(44,44)$ & $(44,44)$ \\
Number of states & 19 & 20 & 20 & 22 & 23 & 27 & 22 & 23 & 21 \\
$D_{1}$ & 118 & 128 & 132 & 140 & 154 & 166 & 142 & 150 & 138 \\
$D_{2}$ & 115 & 128 & 132 & 140 & 154 & 166 & 128 & 132 & 120 \\
\hline \hline
\end{tabular}

the expressions

${ }_{i}\left\langle 1_{k} \mid 1_{k^{\prime}}\right\rangle_{f}=\sum_{p n}\left[X_{k}(i, p n) X_{k^{\prime}}(f, p n)-Y_{k}(i, p n) Y_{k}(f, p n)\right]$.

Throughout this paper, the Rose [21] convention for the Wigner-Eckart theorem is used.

Before closing this section we would like to point out what is specific to our formalism. Since our single-particle states are projected spherical states, the pnQRPA formalism is fully identical to the one usually employed for spherical nuclei. In the vibrational limit $(d \rightarrow 0)$, our basis goes to the spherical shell-model basis; therefore, one might say that the present formalism provides a unified description of spherical and deformed nuclei.

\section{NUMERICAL RESULTS}

The formalism described in the previous section has been applied to the following 10 isotopes: ${ }^{48} \mathrm{Ca},{ }^{96} \mathrm{Zr},{ }^{100} \mathrm{Mo},{ }^{104} \mathrm{Ru}$, ${ }^{110} \mathrm{Pd},{ }^{116} \mathrm{Cd},{ }^{128} \mathrm{Te},{ }^{130} \mathrm{Te},{ }^{134} \mathrm{Xe}$, and ${ }^{136} \mathrm{Xe}$. The spherical shell-model parameters for these double $\beta$ emitters and the corresponding daughter nuclei are given by

$$
\hbar \omega_{0}=41 A^{1 / 3}, \quad C=2 \hbar \omega_{0} \kappa, \quad D=\hbar \omega_{0} \mu,
$$

with the strength parameters $\kappa$ and $\mu$ having the same $(Z, N)$ dependence as in Ref. [22].

The angular-momentum-projected basis depends on two additional parameters: the deformation parameter $d$ and the factor $k$ entering the canonical transformation relating the quadrupole coordinate and boson operators. They are fixed in the same manner as in [15]. Indeed, we require that the relative energy for the states $\left|1 f \frac{7}{2} \frac{7}{2}\right\rangle$ and $\left|1 d \frac{5}{2} \frac{1}{2}\right\rangle$ be equal to that of Nilsson levels with $\Omega=\frac{7}{2}$ and $\Omega=\frac{1}{2}$ in the $N=3$ major shell. Moreover, adding to the mean-field term defined before a $Q Q$ two-body interaction, we require that the lowest root for the charge-conserving QRPA equation be equal to the experimental energy of the lowest $2^{+}$state in the parent nucleus. Throughout this paper, the $M$-degenerate states $\Phi_{n l j}^{I M}$ are denoted by $|n+1 l j I\rangle$.

The BCS calculation has been performed within a restricted single-particle space. Because of the level crossing, the restriction of the single-particle space for deformed nuclei is different from that for spherical nuclei. Indeed, in spherical nuclei the Ikeda sum rule (ISR) is satisfied if two major shells plus the spin-orbit partner of the intruder state are included in the single-particle space. Suppose that the neutron open shell has $N=3$ with the intruder state $|1 g 9 / 2\rangle$ in the standard spherical shell-model picture. In the present formalism, including the spin-orbit partner state $|1 g 7 / 2\rangle$ means that we consider the states $\Phi_{0,4, \frac{7}{2}}^{I M}$ with $I=7 / 2,5 / 2,3 / 2,1 / 2$. However, some of these states are higher in energy than states belonging to the $|2 d 5 / 2 I\rangle$ multiplet. Because such features appear in both the upper part of the major open shell of neutrons and the bottom side of the proton major open shell, we truncated the space by considering an inert $(Z, N)$ core and a number of states lying above the core states. The core and the number of outside states were chosen such that the nonoccupation probabilities for the neglected bottom states as well as the occupation probabilities for the ignored upper states were smaller than 0.01. Of course, the single-particle space for protons and neutrons were the same. Our calculations were performed with the core and number of states given in Table I.

Once the single-particle space is defined, the number of the dipole proton-neutron states can be calculated. Furthermore, the dimensions of the pnQRPA matrices for parent $D_{1}$ and daughter $D_{2}$ nuclei are readily obtained. These dimensions are also given in Table I. It is worth mentioning that using the single-particle spaces given in Table I satisfies the Ikeda sum rule for both the parent and daughter nuclei considered in this paper.

Note that despite the fact that single-particle energies have a deformation dependence, we keep calling a major shell a set of states characterized by the same quantum number $N(=2 n+l)$ plus the states from the shell $N+1$ of maxi$\operatorname{mum} j$.

Single-particle parameters $d$ and $k$ as well as the pairing strengths, fixed so that the mass difference of the neighboring even-even nuclei are reproduced, are listed later in Table III.

Now, let us turn our attention to the proton-neutron dipole interactions. Ref. [11] suggested a simple $A$ dependence for these interaction strengths:

$$
\chi=\frac{5.2}{A^{0.7}} \mathrm{MeV}, \quad \chi_{1}=\frac{0.58}{A^{0.7}} \mathrm{MeV} .
$$

We recall that this $A$ dependence of the proton-neutron $p h$ interaction strengths was obtained by fitting the position of the GT resonance for ${ }^{40} \mathrm{Ca},{ }^{90} \mathrm{Zr}$, and ${ }^{208} \mathrm{~Pb}$. The $p p$ interaction strength given above was obtained by fitting the half-lives for $Z \leqslant 40$ nuclei against the single $\beta^{+}$decay. A certain caution, however, is necessary when these formulas are used, since the $A$ dependence is conditioned by the mass region [23] as well as by the single-particle space [24,25]. For example, in Ref. [26] the GT resonance centroids in ${ }^{128} \mathrm{Te}$ and ${ }^{130} \mathrm{Te}$, located at 13.7 and $14.1 \mathrm{MeV}$, respectively, are reproduced with $\chi$ values equal to 0.157 and $0.16 \mathrm{MeV}$, respectively. These values for $\chi$ 
TABLE II. The experimental and theoretical $\log f t$ values characterizing the $\beta^{+} / E C$ and $\beta^{-}$processes of the intermediate nucleus ground state $\left(1^{+}\right)$. Sources of the experimental data are given in brackets.

\begin{tabular}{lcccc}
\hline \hline $\begin{array}{l}\text { Parent } \\
\text { nucleus }\end{array}$ & $\begin{array}{c}\text { Transition } \\
\log f t\end{array}$ & $\begin{array}{c}\text { Intermediate } \\
\text { nucleus }\end{array}$ & $\begin{array}{c}\text { Transition } \\
\log f t\end{array}$ & $\begin{array}{c}\text { Daughter } \\
\text { nucleus }\end{array}$ \\
\hline${ }^{100} \mathrm{Mo}$ & $\beta^{+} / E C$ & ${ }^{100} \mathrm{Tc}$ & $\stackrel{\beta^{-}}{\rightarrow}$ & ${ }^{100} \mathrm{Ru}$ \\
Exp. & $4.45_{-0.30}^{+0.18}[27]$ & $4.66[28]$ & \\
Th. & 4.61 & 4.66 & \\
${ }^{104} \mathrm{Ru}$ & $\beta^{+} / E C$ & ${ }^{104} \mathrm{Rh}$ & $\stackrel{\beta^{-}}{\longrightarrow}$ & ${ }^{104} \mathrm{Pd}$ \\
Exp. & $4.32[29]$ & & $4.55[29]$ & \\
Th. & 4.20 & & 4.62 & \\
${ }^{110} \mathrm{Pd}$ & $\beta^{+} / E C$ & ${ }^{110} \mathrm{Ag}$ & $\stackrel{\beta^{-}}{\rightarrow}$ & ${ }^{110} \mathrm{Cd}$ \\
Exp. & $4.08[30]$ & & $4.66[30]$ & \\
Th. & 3.86 & & 4.83 & \\
${ }^{116} \mathrm{Cd}$ & $\beta^{+} / E C$ & ${ }^{116} \mathrm{In}$ & $\stackrel{\beta^{-}}{\rightarrow}$ & ${ }^{116} \mathrm{Sn}$ \\
Exp. & $4.39_{-0.15}^{+0.1}[31]$ & & $4.662[32]$ & \\
Th. & 4.05 & & 4.670 & \\
${ }^{128} \mathrm{Te}$ & $\beta^{+} / E C$ & ${ }^{128} \mathrm{I}$ & $\stackrel{\beta^{-}}{\rightarrow}$ & ${ }^{128} \mathrm{Xe}$ \\
Exp. & $5.049[33]$ & & $6.061[34]$ & \\
Th. & 4.930 & & 6.226 & \\
\hline \hline
\end{tabular}

are different from the predictions of Eq. (3.2) corresponding to $A=128$ and $A=130$, respectively. Moreover, as we will see later from Table IV, in the current paper the right positions of these GT resonances are obtained by using $\chi=0.268$ for both isotopes. It is noteworthy that the daughter nuclei involved in a double $\beta$ process are stable against $\beta^{+}$transitions. Therefore $\chi_{1}$ is to be determined either by using information about the half-life of a $\beta^{+}$emitter lying close in the nuclide chart to the daughter nucleus under consideration or by fitting the data for a $(p, n)$ reaction having the daughter as a residual nucleus. Hereafter, the ratio $\chi / \chi_{1}$ is denoted, as usual, by $g_{p p}$.

The procedure adopted to fix the proton-neutron dipole interaction strengths is as follows. Whenever in the intermediate odd-odd nucleus the position of the GT resonance centroid is known, the ph interaction strength is fixed so that the above-mentioned data are reproduced. As shown in Table II, for some of the isotopes considered in the present paper, the $\log f t$ values associated with the $\beta^{+} / E C$ and $\beta^{-}$transitions of the corresponding intermediate nuclei are experimentally known. For these particular cases, $\chi$ and $g_{p p}$ are fixed by fitting the two types of experimental data. The $\log f t$ values were calculated by using the following expression for $f t$ :

$$
f t_{\mp}=\frac{6160}{\left[{ }_{l}\left\langle 1_{1}|| \beta^{ \pm}|| 0\right\rangle_{l} g_{A}\right]^{2}} .
$$

Here $\left|1_{1} M\right\rangle$ denotes the first dipole phonon state in the intermediate odd-odd nucleus while $|0\rangle$ is the pnQRPA ground state. The low index $l$ may take the value $i$ and $f$ depending on whether the end state of the transition is characterizing the double $\beta$ parent or daughter nucleus. Therefore $l=f$ is associated with the single $\beta^{-}$transition, and $l=i$ with the $\beta^{+} / E C$ process. We chose $g_{A}=1.0$ in order to take account of the effect of distant states responsible for the "missing strength" in the giant GT resonance [1]. For ${ }^{48} \mathrm{Ca}$, we considered first $\chi$ and $g_{p p}$ (second row of Table IV) as given by Eq. (3.2). To see the effect of $g_{p p}$ on $M_{\mathrm{GT}}$ we repeated the calculations by keeping the same $\chi$ as before but taking $g_{p p}=0$ (third row of Table IV). It seems that fixing $\chi$ so as to reproduce the GT resonance centroid and taking $g_{p p}=0$ yields a better agreement with the experimental data. This situation is presented in the first row of Table IV. For ${ }^{96} \mathrm{Zr}, \chi$ was fixed by fitting the energy for the GT resonance centroid, while $g_{p p}$ was taken as required by Eq. (3.2). For ${ }^{130} \mathrm{Te}$ we took the same $\chi$ and $g_{p p}$ as for ${ }^{128} \mathrm{Te}$. It is interesting to note that for this value of $\chi$ the position of the GT resonance at $14.1 \mathrm{MeV}$ is nicely reproduced. As for the last two double $\beta$ emitters included in Table IV, data are available neither for the GT resonance nor for the $\log f t$ values characterizing the single $\beta^{-}$and $\beta^{+} / E C$ transitions of the corresponding intermediate odd-odd nuclei. For these isotopes we supposed for $\chi$ and $g_{p p}$ a similar linear $1 / A$ dependence as for ${ }^{130} \mathrm{Te}$.

The strength parameters $\chi$ and $g_{p p}$ determined in the manner just described are collected in Table III. They are also listed for each isotope in the first row of Table IV. These parameters yield double $\beta$ half-lives which are to be compared with the corresponding experimental data. The same parameters are used to calculate the single- $\beta$ strength distributions, shown in Figs. 1-4. However, in order to have a fair comparison of the present results and those of Klapdor et al. [35-37], we give in the second row of Table IV the results obtained with $\chi$ and $\chi_{1}$ given by Eq. (3.2) .

Once the parameters involved in the model Hamiltonian are fixed, the BCS and pnQRPA equations can solved and the results used in Eq. (2.21) to calculate the $M_{\mathrm{GT}}$ amplitude. Furthermore, Eq. (2.20) is used to calculate the half-life of the $2 v \beta \beta$ process. The phase factor $F$ is not dependent on the nuclear state structure and therefore was taken as in Refs. [1,38]. The values for $F$ used in this paper correspond to $g_{A}=1.254$ (see the comments at the end of this section). Results for $M_{\mathrm{GT}}$ and $T_{1 / 2}$ are given in Table IV. Therein one may also find the available experimental data.

Before discussing the results presented in Table IV, we would like to discuss the strength distribution for single $\beta^{-}$ and $\beta^{+}$transitions of parent and daughter nuclei. In Figs. 1-4 the strengths $B^{\prime}(G T)_{-}$and $B^{\prime}(G T)_{+}$for parent and daughter nuclei, respectively, folded with a Gaussian having the width equal to $1 \mathrm{MeV}$, are plotted as functions of pnQRPA energies. These are equal to one third of the $\beta^{-}$and $\beta^{+}$strengths in the standard definition. Thus, the difference between the total strengths $B^{\prime}(G T)_{-}$and $B^{\prime}(G T)_{+}$, characterizing the parent nucleus, is to be compared with the sum rule $(N-Z)$. The results of our calculations are to be compared with the available data for the GT giant resonance and single- $\beta$ strengths given in Refs. [26,50,51]. At a glance, one may see that while for Te and $\mathrm{Xe}$ isotopes most of the strength is concentrated in a narrow resonance, for the remaining nuclei the GT resonances have a complex structure spread over a large energy interval. Actually this feature agrees with experimental data showing in ${ }^{128} \mathrm{I}$ and ${ }^{130} \mathrm{I}$ a single peak at 13.7 and $14.1 \mathrm{MeV}$, respectively [26], 
TABLE III. Pairing and Gamow-Teller ph interaction strengths in units of MeV. Ratios of the two dipole interaction (particle-hole and particle-particle) strengths are denoted by $g_{p p}$. Deformation parameters $d$ and factors $k$ of the transformation (2.2) are also presented. The manner in which these parameters were fixed is explained in the text.

\begin{tabular}{|c|c|c|c|c|c|c|c|}
\hline Nucleus & $d$ & $k$ & $G_{p}(\mathrm{MeV})$ & $G_{n}(\mathrm{MeV})$ & $\chi(\mathrm{MeV})$ & $g_{\mathrm{pp}}$ & $\left(\frac{1}{2} Q_{\beta \beta}+m_{e} c^{2}\right)(\mathrm{MeV})$ \\
\hline${ }^{48} \mathrm{Ca}$ & 0.3 & 10.00 & 0.65 & 0.45 & 0.180 & 0.0 & 2.646 \\
\hline${ }^{48} \mathrm{Ti}$ & 0.05 & 2.00 & 0.46 & 0.36 & 0.180 & 0.0 & \\
\hline${ }^{96} \mathrm{Zr}$ & 1.5 & 10.20 & 0.26 & 0.26 & 0.5 & 0.112 & 2.186 \\
\hline${ }^{96} \mathrm{Mo}$ & 1.2 & 7.20 & 0.3 & 0.3 & 0.5 & 0.112 & \\
\hline${ }^{100} \mathrm{Mo}$ & -1.4 & 10.00 & 0.28 & 0.26 & 0.060 & 1.600 & 2.026 \\
\hline${ }^{100} \mathrm{Ru}$ & -0.6 & 3.6 & 0.285 & 0.220 & 0.060 & 1.600 & \\
\hline${ }^{104} \mathrm{Ru}$ & -1.55 & 8.80 & 0.26 & 0.2 & 0.150 & 2.750 & 1.161 \\
\hline${ }^{104} \mathrm{Pd}$ & -1.35 & 6.94 & 0.26 & 0.180 & 0.150 & 2.750 & \\
\hline${ }^{110} \mathrm{Pd}$ & -1.6 & 6.00 & 0.30 & 0.32 & 0.148 & 2.450 & 1.516 \\
\hline${ }^{110} \mathrm{Cd}$ & -0.8 & 3.06 & 0.30 & 0.18 & 0.148 & 2.450 & \\
\hline${ }^{116} \mathrm{Cd}$ & -1.8 & 3.00 & 0.20 & 0.245 & 0.238 & 1.680 & 1.916 \\
\hline${ }^{116} \mathrm{Sn}$ & -1.2 & 2.50 & 0.18 & 0.275 & 0.238 & 1.680 & \\
\hline${ }^{128} \mathrm{Te}$ & 0.5 & 1.62 & 0.27 & 0.220 & 0.268 & 1.250 & 0.946 \\
\hline${ }^{128} \mathrm{Xe}$ & 1.7 & 6.50 & 0.23 & 0.220 & 0.268 & 1.250 & \\
\hline${ }^{130} \mathrm{Te}$ & 0.493 & 1.88 & 0.24 & 0.210 & 0.268 & 1.300 & 1.776 \\
\hline${ }^{130} \mathrm{Xe}$ & 1.4 & 5.00 & 0.24 & 0.205 & 0.268 & 1.300 & \\
\hline${ }^{134} \mathrm{Xe}$ & -0.1 & 1.95 & 0.28 & 0.300 & 0.260 & 1.261 & 0.931 \\
\hline${ }^{134} \mathrm{Ba}$ & -0.468 & 1.50 & 0.24 & 0.240 & 0.260 & 1.261 & \\
\hline${ }^{136} \mathrm{Xe}$ & -0.1 & 1.80 & 0.23 & 0.29 & 0.256 & 1.243 & 1.751 \\
\hline${ }^{136} \mathrm{Ba}$ & -0.698 & 2.16 & 0.19 & 0.20 & 0.256 & 1.243 & \\
\hline
\end{tabular}

TABLE IV. The Gamow-Teller amplitude for the $2 \nu \beta \beta$ decay, in units of $\mathrm{MeV}^{-1}$, and the corresponding half-life $\left(T_{1 / 2}\right)$ in years are listed for 10 ground-to-ground transitions. The sources of experimental and theoretical half-lives are given in brackets.

\begin{tabular}{|c|c|c|c|c|c|c|c|}
\hline \multirow[t]{2}{*}{$2 \nu \beta \beta$ decay } & \multirow{2}{*}{$\begin{array}{c}\chi \\
(\mathrm{MeV})\end{array}$} & \multirow[t]{2}{*}{$g_{p p}$} & \multirow{2}{*}{$\begin{array}{c}\left|M_{\mathrm{GT}}\right| \\
\left(\mathrm{MeV}^{-1}\right)\end{array}$} & \multicolumn{4}{|c|}{$T_{1 / 2}(\mathrm{yr})$} \\
\hline & & & & Present & Exp. & Suhonen et al. & Klapdor et al. \\
\hline${ }^{48} \mathrm{Ca} \rightarrow{ }^{48} \mathrm{Ti}$ & $\begin{array}{l}0.180 \\
0.346 \\
0.346\end{array}$ & $\begin{array}{l}0.0 \\
0.112 \\
0.0\end{array}$ & $\begin{array}{l}0.043 \\
0.032 \\
0.036\end{array}$ & $\begin{array}{l}5.23 \times 10^{19} \\
9.27 \times 10^{19} \\
7.48 \times 10^{19}\end{array}$ & $(4.2 \pm 1.2) \times 10^{19}[39]$ & & $3.2 \times 10^{19}[37]$ \\
\hline${ }^{96} \mathrm{Zr} \rightarrow{ }^{96} \mathrm{Mo}$ & $\begin{array}{l}0.500 \\
0.213\end{array}$ & $\begin{array}{l}0.112 \\
0.112\end{array}$ & $\begin{array}{l}0.113 \\
0.219\end{array}$ & $\begin{array}{l}1.66 \times 10^{19} \\
0.44 \times 10^{19}\end{array}$ & $\left(1.4_{-0.5}^{+3.5}\right) \times 10^{19}[39]$ & $0.44 \times 10^{20}[1]$ & $5.2 \times 10^{17}[35]$ \\
\hline${ }^{100} \mathrm{Mo} \rightarrow{ }^{100} \mathrm{Ru}$ & $\begin{array}{l}0.060 \\
0.207\end{array}$ & $\begin{array}{l}1.600 \\
0.112\end{array}$ & $\begin{array}{l}0.305 \\
0.212\end{array}$ & $\begin{array}{l}4.61 \times 10^{18} \\
9.55 \times 10^{18}\end{array}$ & $\begin{array}{c}(8.0 \pm 0.6) \times 10^{18}[39] \\
\left(0.115_{-0.02}^{+0.03}\right) \times 10^{20}[40] \\
\left(0.033_{-0.01}^{+0.02}\right) \times 10^{20}[41,42]\end{array}$ & $2.9 \times 10^{18}[45]$ & $1.8 \times 10^{18}[35]$ \\
\hline${ }^{104} \mathrm{Ru} \rightarrow{ }^{104} \mathrm{Pd}$ & $\begin{array}{l}0.150 \\
0.201\end{array}$ & $\begin{array}{l}2.750 \\
0.112\end{array}$ & $\begin{array}{l}0.781 \\
0.343\end{array}$ & $\begin{array}{l}0.76 \times 10^{21} \\
3.95 \times 10^{21}\end{array}$ & & & $\begin{array}{r}1.8 \times 10^{21}[35] \\
3.09 \times 10^{22}[36]\end{array}$ \\
\hline${ }^{110} \mathrm{Pd} \rightarrow{ }^{110} \mathrm{Cd}$ & $\begin{array}{l}0.148 \\
0.194\end{array}$ & $\begin{array}{l}2.45 \\
0.112\end{array}$ & $\begin{array}{l}0.263 \\
0.218\end{array}$ & $\begin{array}{l}15.85 \times 10^{19} \\
22.99 \times 10^{19}\end{array}$ & & & $\begin{array}{r}5.0 \times 10^{19}[35] \\
1.24 \times 10^{21}[36]\end{array}$ \\
\hline${ }^{116} \mathrm{Cd} \rightarrow{ }^{116} \mathrm{Sn}$ & $\begin{array}{l}0.238 \\
0.187\end{array}$ & $\begin{array}{l}1.680 \\
0.112\end{array}$ & $\begin{array}{l}0.116 \\
0.069\end{array}$ & $\begin{array}{r}3.86 \times 10^{19} \\
10.96 \times 10^{19}\end{array}$ & $(3.2 \pm 0.3) \times 10^{19}[39]$ & $5.1 \times 10^{19}[46]$ & $\begin{array}{r}8.3 \times 10^{18}[35] \\
3.75 \times 10^{19}[47]\end{array}$ \\
\hline${ }^{128} \mathrm{Te} \rightarrow{ }^{128} \mathrm{Xe}$ & $\begin{array}{l}0.268 \\
0.174\end{array}$ & $\begin{array}{l}1.250 \\
0.112\end{array}$ & $\begin{array}{l}0.090 \\
0.127\end{array}$ & $\begin{array}{l}0.55 \times 10^{24} \\
0.28 \times 10^{24}\end{array}$ & $\begin{array}{l}(7.2 \pm 0.3) \times 10^{24}[39] \\
(1.5 \pm 0.2) \times 10^{24}[43]\end{array}$ & $5.6 \times 10^{23}[46]$ & $\begin{array}{l}1.2 \times 10^{23}[35] \\
5.7 \times 10^{23}[47,48]\end{array}$ \\
\hline${ }^{130} \mathrm{Te} \rightarrow{ }^{130} \mathrm{Xe}$ & $\begin{array}{l}0.268 \\
0.172\end{array}$ & $\begin{array}{l}1.300 \\
0.112\end{array}$ & $\begin{array}{l}0.055 \\
0.091\end{array}$ & $\begin{array}{l}0.261 \times 10^{21} \\
0.097 \times 10^{21}\end{array}$ & $\begin{array}{c}(1.5-2.8) \times 10^{21}[40] \\
(2.7 \pm 0.1) \times 10^{21}[39] \\
(0.75 \pm 0.3) \times 10^{21}[44]\end{array}$ & $0.26 \times 10^{21}[46]$ & $\begin{array}{l}1.9 \times 10^{19}[35] \\
1.2 \times 10^{20}[47,48]\end{array}$ \\
\hline${ }^{134} \mathrm{Xe} \rightarrow{ }^{134} \mathrm{Ba}$ & $\begin{array}{l}0.260 \\
0.169\end{array}$ & $\begin{array}{l}1.261 \\
0.112\end{array}$ & $\begin{array}{l}0.039 \\
0.040\end{array}$ & $\begin{array}{l}3.75 \times 10^{24} \\
3.49 \times 10^{24}\end{array}$ & & & $\begin{array}{l}5.1 \times 10^{22}[35] \\
2.5 \times 10^{23}[47,48]\end{array}$ \\
\hline${ }^{136} \mathrm{Xe} \rightarrow{ }^{136} \mathrm{Ba}$ & $\begin{array}{l}0.256 \\
0.167\end{array}$ & $\begin{array}{l}1.243 \\
0.112\end{array}$ & $\begin{array}{l}0.039 \\
0.068\end{array}$ & $\begin{array}{r}5.102 \times 10^{20} \\
1.69 \times 10^{20}\end{array}$ & $>8.1 \times 10^{20}[39]$ & $1.3 \times 10^{20}[46]$ & $\begin{array}{l}6.0 \times 10^{19}[35] \\
3.3 \times 10^{19}[47,48]\end{array}$ \\
\hline
\end{tabular}



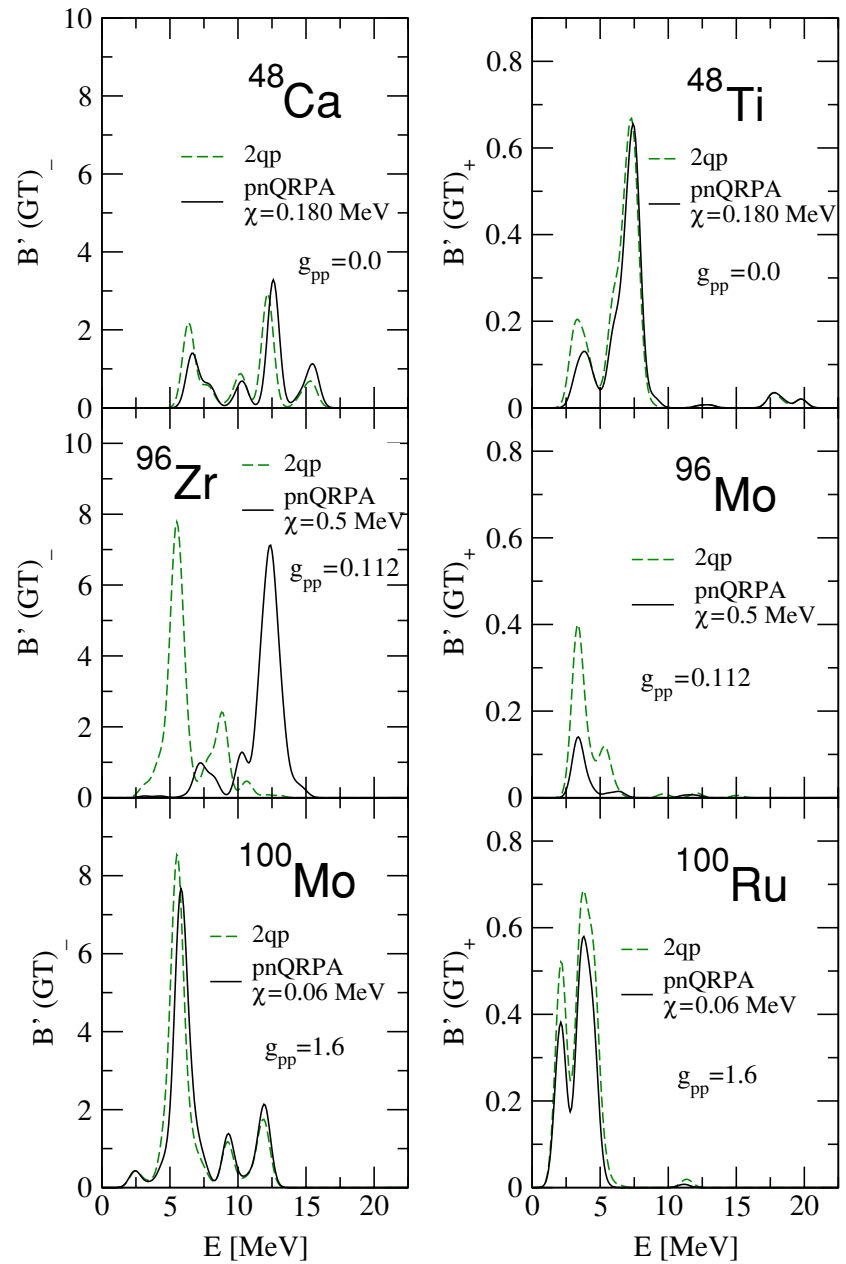

FIG. 1. (Color online) Single $\beta^{-}$strengths (left panel) and single $\beta^{+}$strengths (right panel), folded with a Gaussian function having the width of $1 \mathrm{MeV}$, are plotted as functions of the energy within the BCS and pnQRPA approximations. The pnQRPA calculations correspond to the values of $\chi$ and $g_{p p}$ listed inside the graphs.

while in ${ }^{100} \mathrm{Tc}$ and ${ }^{116} \mathrm{In}[50]$ two peaks show at $(7.8,13.2)$ and $(8.8,14.30) \mathrm{MeV}$. The locations of these peaks are reasonably well reproduced. In the case of ${ }^{100} \mathrm{Mo}$, the first peak is higher than the one centered at $12.3 \mathrm{MeV}$. Increasing the value of the repulsive $p h$ interaction $\chi$ changes the ordering of the two peak magnitudes.

The $\beta^{-}$and $\beta^{+}$strengths of ${ }^{48} \mathrm{Ca}$ have been studied in Refs. [51] and [50]. Thus, the GT resonance has been populated in the reaction ${ }^{48} \mathrm{Ca}(p, n)^{48} \mathrm{Sc}$. This resonance is spread over an energy interval between 4.5 and $14.5 \mathrm{MeV}$. The result
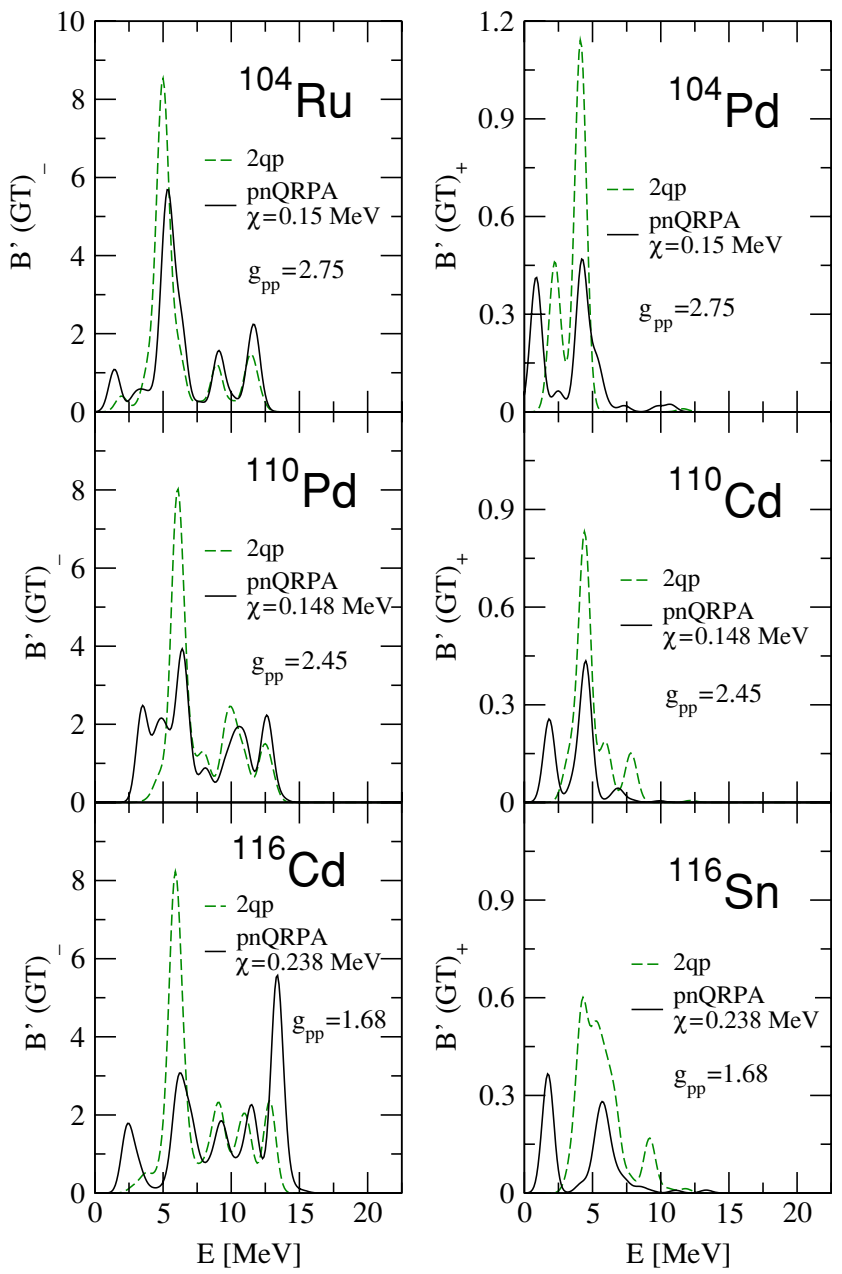

FIG. 2. (Color online) As in Fig. 1 for six more isotopes.

presented for $B^{\prime}(G T)_{-}$in Fig. 1, upper left panel, agrees with the experimental data. The total $\beta^{-}$strength quenched with a factor of 0.6 [52], accounting for the polarization effects on the single- $\beta$ transition operator, ignored in the present paper, is compared with the corresponding data in Table V. As shown therein, the agreement between the calculated strength and the corresponding data is reasonably good. The only known data for the total $\beta^{+}$GT strength is for ${ }^{48} \mathrm{Ti}$ :

$$
\sum B(G T)_{+}=1.42 \pm 0.2 \text {. }
$$

Our calculations, corresponding to the first row of Table IV, predict for this strength the value 2.59 .

Comparing the $\beta^{-}$strength distribution among $2 q p$ states with those corresponding to pnQRPA states, one may conclude

TABLE V. Total strengths for the Gamow-Teller $\beta^{-}$transition (first row) are compared with the available experimental data (second row). Theoretical results are quenched with a factor of 0.6. Data for ${ }^{100} \mathrm{Mo}$ and ${ }^{116} \mathrm{Cd}$ are from Ref. [50] while those for ${ }^{128,130} \mathrm{Te}$ are from Ref. [26].

\begin{tabular}{lcccccccccc}
\hline \hline Nucleus & ${ }^{48} \mathrm{Ca}$ & ${ }^{96} \mathrm{Zr}$ & ${ }^{100} \mathrm{Mo}$ & ${ }^{104} \mathrm{Ru}$ & ${ }^{110} \mathrm{Pd}$ & ${ }^{116} \mathrm{Cd}$ & ${ }^{128} \mathrm{Te}$ & ${ }^{130} \mathrm{Te}$ & ${ }^{134} \mathrm{Xe}$ & ${ }^{136} \mathrm{Xe}$ \\
\hline $0.6 \sum B(G T)_{-}$ & 15.650 & 28.886 & 30.040 & 29.527 & 33.172 & 38.051 & 43.340 & 47.059 & 47.028 & 50.703 \\
$\sum B(G T)_{-}^{\exp }$ & - & - & 26.690 & - & - & 32.700 & 40.080 & 45.900 & - & - \\
\hline \hline
\end{tabular}



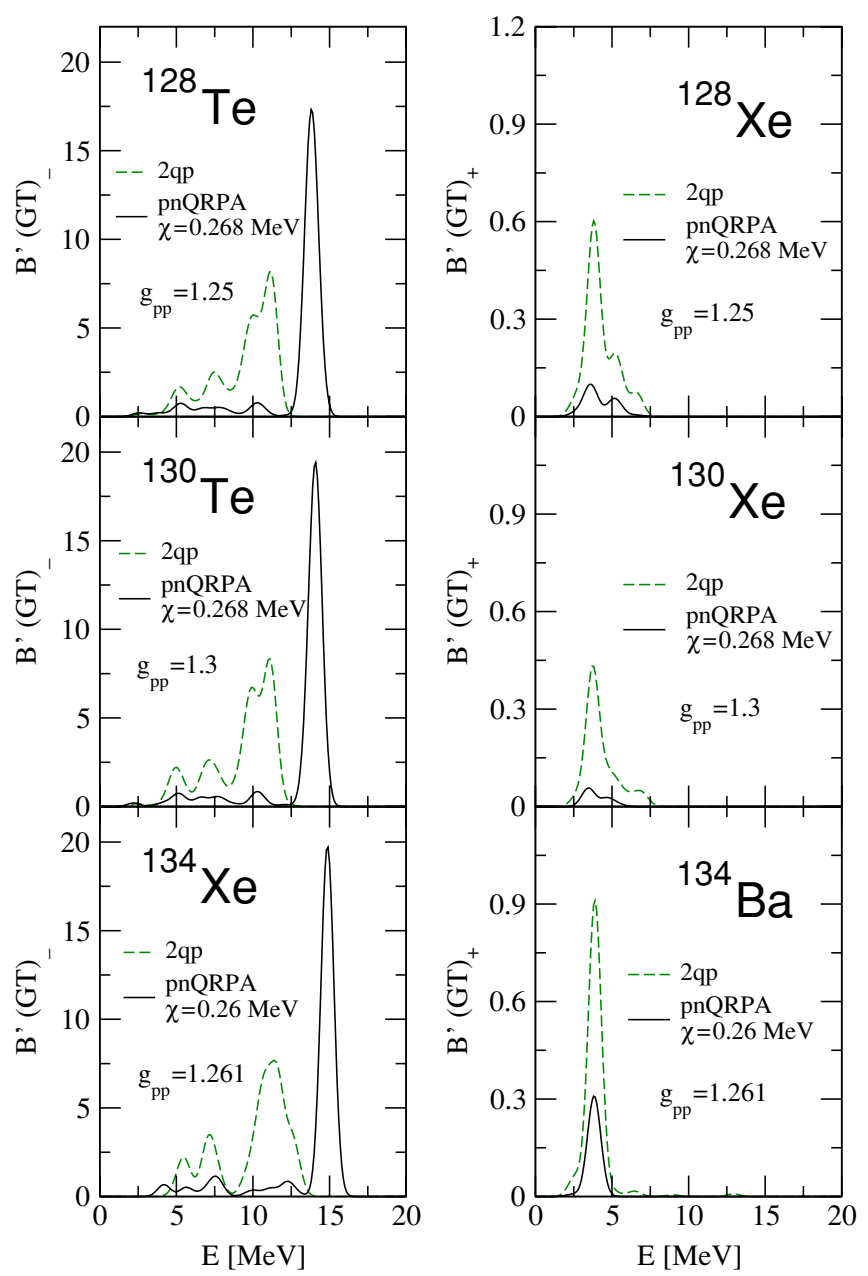

FIG. 3. (Color online) As in Fig. 1 for six more isotopes.

that the quasiparticle correlations accounted for by the pnQRPA approach favor the displacement of the strength toward higher energies. This, in fact, is due to the repulsive character of the ph interaction. As shown in Figs. 1-4, this effect is more pronounced for ${ }^{96} \mathrm{Zr},{ }^{128,130} \mathrm{Te}$, and ${ }^{134,136} \mathrm{Xe}$.
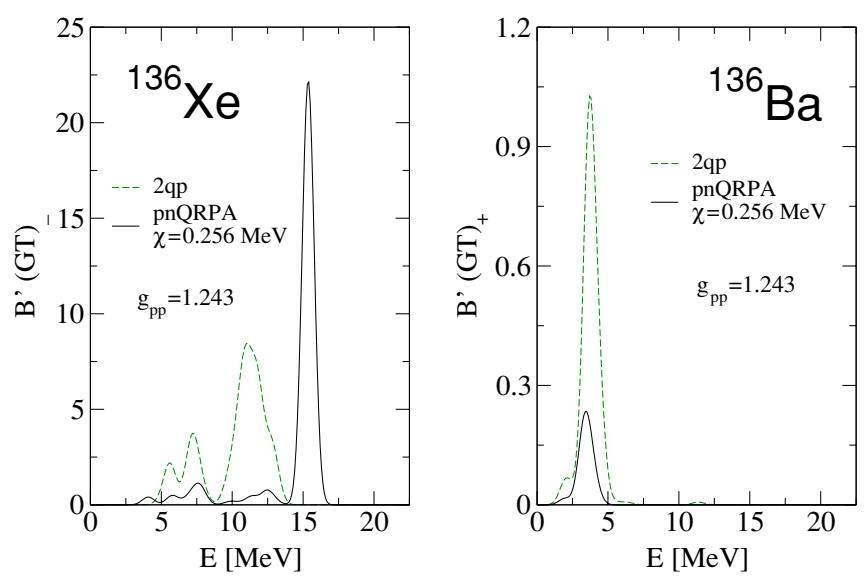

FIG. 4. (Color online) As in Fig. 1 for two more isotopes.

In Table VI, we see that most of the $\beta^{-}$strengths are due to the transitions relating to the proton and neutron $g$ states. In contrast, in ${ }^{48} \mathrm{Ca},{ }^{96} \mathrm{Zr}$, and ${ }^{116} \mathrm{Cd}$, the single-particle decays involving $f$ states carry most of the transition strength. Also the GT resonance peak in ${ }^{130} \mathrm{Te}$ is determined mainly by the transition in the $2 d$ state. In the lighter isotopes the transitions $\nu I \rightarrow \pi I^{\prime}$ where either $I$ or $I^{\prime}$ are equal to $\frac{1}{2}$ or $\frac{3}{2}$ prevail; in Te and Xe isotopes the transitions $\nu \frac{7}{2} \rightarrow \pi \frac{5}{2}$ and $\nu \frac{5}{2} \rightarrow \pi \frac{7}{2}$ are dominant.

Concerning the $\beta^{+}$strength distribution shown in Figs. 1-4, right panels, the following features are to be noticed. The magnitude of this strength is much smaller than that of $\beta^{-}$shown in the left panels. Moreover, the final states in the $\beta^{+}$process are lying in the lower part of the spectrum, below 7.5 MeV. This suggests that the $p p$ interaction may strongly influence the strength distribution among these states. The sensitivity of the $\beta^{+}$decay rate against the $p p$ interaction was first noticed in Ref. [53]. Due to this feature the $\beta \beta$ transition amplitude is also significantly affected by increasing $g_{p p}$. Since the $p h$ and $p p$ interactions have different natures, one is repulsive and the other attractive, one expects that the two interactions have opposite effects on the $\beta^{+}$strength. When the $p p$ interaction is large compared to the $p h$ interaction, the $\beta^{+}$strength in the quasiparticle picture is shifted toward the lower states. Such is the case for ${ }^{104} \mathrm{Pd},{ }^{110} \mathrm{Cd}$, and ${ }^{116} \mathrm{Sn}$. When both the $p h$ and $p p$ interactions are large, the $\beta^{+}$strength of $2 q p$ states are very much suppressed in the pnQRPA approach. Such situations are found in ${ }^{128,130} \mathrm{Xe}$ and ${ }^{134,136} \mathrm{Ba}$.

By inspecting Table VII, we conclude that the largest $\beta^{+}$strength is carried by the single particle proton-neutron transition in the shells $1 g$ (for ${ }^{100} \mathrm{Ru},{ }^{104} \mathrm{Pd},{ }^{116} \mathrm{Sn},{ }^{128} \mathrm{Xe}$, ${ }^{136} \mathrm{Ba}$ ), $2 d$ (for ${ }^{96} \mathrm{Mo},{ }^{110} \mathrm{Cd}$ ), $1 f$ (for ${ }^{48} \mathrm{Ti}$ ), $2 f$ (for ${ }^{134} \mathrm{Ba}$ ), and $1 h$ (for ${ }^{130} \mathrm{Xe}$ ). Identifying the common $2 q p$ configurations carrying most efficiently the $\beta^{-}$and $\beta^{+}$strengths from Tables VIII and IX, respectively, one may determine which pnQRPA states excited from the parent and daughter ground state, maximally overlap each other and therefore bring a large contribution to the double $\beta$ decay. In some of the depicted cases, the state excited by the $\beta^{-}$transition operator belongs to the GT resonance. An excellent example is the transition ${ }^{48} \mathrm{Ca} \rightarrow{ }^{48} \mathrm{Ti}$, where the state at 10.326 excited from the ground state of ${ }^{48} \mathrm{Ca}$ and the state with energy of 5.957 excited from ${ }^{48} \mathrm{Ti}$ have maximal overlap because of the $2 q p$ state $\pi\left(3 p \frac{1}{2} \frac{1}{2}\right), v\left(3 p \frac{3}{2} \frac{3}{2}\right)$ which, carries a large strength in both $\beta^{-}$and $\beta^{+}$transitions.

Let us now focus our attention on the GT double $\beta$ transition amplitude. This was calculated by means of Eq. (2.21), where the energy shifts (2.19) are those listed in Table III and the measured values for $1^{+}$are collected in Table X. The states, energies, and overlap matrix elements involved in the $M_{\mathrm{GT}}$ expression were calculated within the pnQRPA approach. The results corresponding to various sets of proton-neutron dipole interactions, fixed in the manner explained before, are listed in Table IV, which also gives, the half-lives of the $2 \nu \beta \beta$ process. The agreement with the available data is fairly good.

Comparing our results with those of Klapdor et al. [35, $36,48]$, one may say that the half-lives predicted by the present paper with the dipole interaction strengths given by 
TABLE VI. The strengths carried by the pnQRPA states contributing to the first, second, and third (if any) peaks from the left panels of Figs. 1-4. To the left of each number, the 2qp configuration closest in energy to the corresponding pnQRPA state is given. This is the dominant configuration of the chosen $p n$ phonon state. The states $\Phi_{n l j}^{I M}$ [see Eq. (2.6)] are specified by the quantum numbers $(N l j I)$, where $N=2 n+1$. Also, the orbital angular momentum values $0,1,2, \ldots$ are mentioned by the letters $s, p, d, \ldots$, respectively.

\begin{tabular}{|c|c|c|c|c|c|c|}
\hline \multirow[t]{2}{*}{ Nucleus } & \multicolumn{2}{|l|}{1 st peak } & \multicolumn{2}{|l|}{ 2nd peak } & \multicolumn{2}{|l|}{ 3rd peak } \\
\hline & Transition & Strength & Transition & Strength & Transition & Strength \\
\hline \multirow[t]{2}{*}{${ }^{48} \mathrm{Ca}$} & $v\left(3 f \frac{7}{2} \frac{5}{2}\right) \rightarrow \pi\left(3 f \frac{7}{2} \frac{7}{2}\right)$ & 1.155 & $v\left(3 p \frac{3}{2} \frac{3}{2}\right) \rightarrow \pi\left(3 p \frac{3}{2} \frac{1}{2}\right)$ & 0.519 & $v\left(3 p \frac{3}{2} \frac{3}{2}\right) \rightarrow \pi\left(3 p \frac{1}{2} \frac{1}{2}\right)$ & 0.700 \\
\hline & & & & & $v\left(3 f \frac{5}{2} \frac{3}{2}\right) \rightarrow \pi\left(3 f \frac{7}{2} \frac{1}{2}\right)$ & 3.344 \\
\hline \multirow[t]{3}{*}{${ }^{96} \mathrm{Zr}$} & $v\left(3 f \frac{5}{2} \frac{5}{2}\right) \rightarrow \pi\left(3 f \frac{5}{2} \frac{3}{2}\right)$ & 0.602 & $v\left(3 f \frac{7}{2} \frac{7}{2}\right) \rightarrow \pi\left(3 p \frac{5}{2} \frac{5}{2}\right)$ & 1.040 & $v\left(3 f \frac{5}{2} \frac{5}{2} \rightarrow \pi\left(3 f \frac{7}{2} \frac{3}{2}\right)\right.$ & 5.842 \\
\hline & $v\left(4 d \frac{5}{2} \frac{3}{2}\right) \rightarrow \pi\left(4 d \frac{5}{2} \frac{5}{2}\right)$ & 0.467 & $v\left(3 f \frac{5}{2} \frac{5}{2}\right) \rightarrow \pi\left(3 f \frac{7}{2} \frac{5}{2}\right)$ & 2.473 & $v\left(3 f \frac{7}{2} \frac{5}{2}\right) \rightarrow \pi\left(3 f \frac{5}{2} \frac{3}{2}\right)$ & 2.212 \\
\hline & & & $v\left(3 p \frac{1}{2} \frac{1}{2}\right) \rightarrow \pi\left(3 p \frac{3}{2} \frac{3}{2}\right)$ & 0.786 & & \\
\hline \multirow[t]{3}{*}{${ }^{100} \mathrm{Mo}$} & $v\left(4 g \frac{9}{2} \frac{7}{2}\right) \rightarrow \pi\left(4 g \frac{9}{2} \frac{9}{2}\right)$ & 4.950 & $v\left(4 d \frac{5}{2} \frac{5}{2}\right) \rightarrow \pi\left(4 d \frac{3}{2} \frac{3}{2}\right)$ & 1.456 & $v\left(4 g \frac{9}{2} \frac{9}{2}\right) \rightarrow \pi\left(4 g \frac{7}{2} \frac{7}{2}\right)$ & 0.702 \\
\hline & $v\left(4 g \frac{7}{2} \frac{7}{2}\right) \rightarrow \pi\left(4 g \frac{9}{2} \frac{9}{2}\right)$ & 0.428 & & & $v\left(3 f \frac{7}{2} \frac{3}{2}\right) \rightarrow \pi\left(3 f \frac{5}{2} \frac{1}{2}\right)$ & 0.716 \\
\hline & $v\left(4 d \frac{5}{2} \frac{3}{2}\right) \rightarrow \pi\left(4 d \frac{5}{2} \frac{1}{2}\right)$ & 1.005 & & & & \\
\hline \multirow[t]{3}{*}{${ }^{104} \mathrm{Ru}$} & $v\left(4 d \frac{3}{2} \frac{3}{2}\right) \rightarrow \pi\left(4 d \frac{5}{2} \frac{1}{2}\right)$ & 1.342 & $v\left(4 g \frac{9}{2} \frac{3}{2}\right) \rightarrow \pi\left(4 g \frac{7}{2} \frac{5}{2}\right)$ & 0.885 & $v\left(4 g \frac{9}{2} \frac{9}{2}\right) \rightarrow \pi\left(4 g \frac{7}{2} \frac{7}{2}\right)$ & 0.563 \\
\hline & $v\left(4 g \frac{9}{2} \frac{3}{2}\right) \rightarrow \pi\left(4 g \frac{9}{2} \frac{1}{2}\right)$ & 2.722 & $v\left(4 g \frac{9}{2} \frac{7}{2}\right) \rightarrow \pi\left(4 g \frac{7}{2} \frac{7}{2}\right)$ & 0.400 & $v\left(3 f \frac{5}{2} \frac{1}{2}\right) \rightarrow \pi\left(3 f \frac{7}{2} \frac{3}{2}\right)$ & 1.703 \\
\hline & $v\left(5 h \frac{11}{2} \frac{11}{2}\right) \rightarrow \pi\left(5 h \frac{11}{2} \frac{11}{2}\right)$ & 1.321 & & & $v\left(3 f \frac{7}{2} \frac{3}{2}\right) \rightarrow \pi\left(3 f \frac{5}{2} \frac{1}{2}\right)$ & 0.266 \\
\hline \multirow[t]{3}{*}{${ }^{110} \mathrm{Pd}$} & $v\left(4 g \frac{7}{2} \frac{5}{2}\right) \rightarrow \pi\left(4 g \frac{9}{2} \frac{5}{2}\right)$ & 2.554 & $v\left(4 g \frac{9}{2} \frac{5}{2}\right) \rightarrow \pi\left(4 g \frac{7}{2} \frac{5}{2}\right)$ & 1.028 & $v\left(4 g \frac{9}{2} \frac{7}{2}\right) \rightarrow \pi\left(4 g \frac{7}{2} \frac{5}{2}\right)$ & 1.570 \\
\hline & $\nu\left(4 g \frac{7}{2} \frac{5}{2}\right) \rightarrow \pi\left(4 g \frac{9}{2} \frac{3}{2}\right)$ & 1.080 & $v\left(4 d \frac{3}{2} \frac{1}{2}\right) \rightarrow \pi\left(4 d \frac{3}{2} \frac{1}{2}\right)$ & 1.171 & $v\left(3 f \frac{5}{2} \frac{3}{2}\right) \rightarrow \pi\left(3 f \frac{7}{2} \frac{1}{2}\right)$ & 0.294 \\
\hline & $\nu\left(4 g \frac{9}{2} \frac{5}{2}\right) \rightarrow \pi\left(4 g \frac{9}{2} \frac{7}{2}\right)$ & 2.705 & & & & \\
\hline \multirow[t]{3}{*}{${ }^{116} \mathrm{Cd}$} & $v\left(4 g \frac{7}{2} \frac{5}{2}\right) \rightarrow \pi\left(4 g \frac{7}{2} \frac{7}{2}\right)$ & 1.668 & $v\left(4 g \frac{9}{2} \frac{5}{2}\right) \rightarrow \pi\left(4 g \frac{7}{2} \frac{7}{2}\right)$ & 0.769 & $v\left(3 f \frac{7}{2} \frac{3}{2}\right) \rightarrow \pi\left(3 f \frac{5}{2} \frac{1}{2}\right)$ & 1.121 \\
\hline & $v\left(4 d \frac{5}{2} \frac{1}{2}\right) \rightarrow \pi\left(4 d \frac{3}{2} \frac{3}{2}\right)$ & 0.462 & $\nu\left(4 g \frac{7}{2} \frac{7}{2}\right) \rightarrow \pi\left(4 g \frac{9}{2} \frac{5}{2}\right)$ & 0.927 & $v\left(3 f \frac{5}{2} \frac{3}{2}\right) \rightarrow \pi\left(3 f \frac{7}{2} \frac{1}{2}\right)$ & 2.647 \\
\hline & & & $v\left(4 g \frac{7}{2} \frac{3}{2}\right) \rightarrow \pi\left(4 g \frac{9}{2} \frac{1}{2}\right)$ & 1.247 & $v\left(3 f \frac{7}{2} \frac{3}{2}\right) \rightarrow \pi\left(3 f \frac{5}{2} \frac{3}{2}\right)$ & 1.971 \\
\hline \multirow[t]{2}{*}{${ }^{128} \mathrm{Te}$} & $v\left(4 g \frac{7}{2} \frac{1}{2}\right) \rightarrow \pi\left(4 g \frac{7}{2} \frac{3}{2}\right)$ & 0.446 & $v\left(4 g \frac{9}{2} \frac{5}{2}\right) \rightarrow \pi\left(4 g \frac{7}{2} \frac{3}{2}\right)$ & 0.467 & $v\left(4 g \frac{9}{2} \frac{5}{2}\right) \rightarrow \pi\left(4 g \frac{7}{2} \frac{7}{2}\right)$ & 12.483 \\
\hline & $v\left(4 g \frac{7}{2} \frac{3}{2}\right) \rightarrow \pi\left(4 g \frac{9}{2} \frac{5}{2}\right)$ & 0.380 & $v\left(4 g \frac{9}{2} \frac{3}{2}\right) \rightarrow \pi\left(4 g \frac{7}{2} \frac{1}{2}\right)$ & 0.198 & $v\left(4 d \frac{5}{2} \frac{3}{2}\right) \rightarrow \pi\left(4 d \frac{3}{2} \frac{3}{2}\right)$ & 7.316 \\
\hline \multirow[t]{2}{*}{${ }^{130} \mathrm{Te}$} & $v\left(4 g \frac{7}{2} \frac{5}{2}\right) \rightarrow \pi\left(4 g \frac{7}{2} \frac{7}{2}\right)$ & 0.462 & $v\left(5 h \frac{11}{2} \frac{1}{2}\right) \rightarrow \pi\left(5 h \frac{9}{2} \frac{3}{2}\right)$ & 0.430 & $v\left(4 g \frac{9}{2} \frac{5}{2}\right) \rightarrow \pi\left(4 g \frac{7}{2} \frac{7}{2}\right)$ & 2.543 \\
\hline & $v\left(4 d \frac{3}{2} \frac{1}{2}\right) \rightarrow \pi\left(4 d \frac{5}{2} \frac{3}{2}\right)$ & 0.342 & $v\left(4 g \frac{9}{2} \frac{3}{2}\right) \rightarrow \pi\left(4 g \frac{7}{2} \frac{1}{2}\right)$ & 0.291 & $v\left(4 d \frac{5}{2} \frac{3}{2}\right) \rightarrow \pi\left(4 d \frac{3}{2} \frac{3}{2}\right)$ & 19.205 \\
\hline \multirow[t]{2}{*}{${ }^{134} \mathrm{Xe}$} & $v\left(5 h \frac{11}{2} \frac{9}{2}\right) \rightarrow \pi\left(5 h \frac{11}{2} \frac{11}{2}\right)$ & 0.713 & $v\left(4 g \frac{7}{2} \frac{5}{2}\right) \rightarrow \pi\left(4 g \frac{9}{2} \frac{5}{2}\right)$ & 0.659 & $v\left(4 d \frac{5}{2} \frac{1}{2}\right) \rightarrow \pi\left(4 d \frac{3}{2} \frac{1}{2}\right)$ & 0.784 \\
\hline & & & $v\left(4 d \frac{3}{2} \frac{1}{2}\right) \rightarrow \pi\left(4 d \frac{3}{2} \frac{3}{2}\right)$ & 0.625 & $v\left(4 g \frac{9}{2} \frac{7}{2}\right) \rightarrow \pi\left(4 g \frac{9}{2} \frac{5}{2}\right)$ & 21.050 \\
\hline \multirow[t]{2}{*}{${ }^{136} \mathrm{Xe}$} & $v\left(4 g \frac{7}{2} \frac{3}{2}\right) \rightarrow \pi\left(4 g \frac{9}{2} \frac{5}{2}\right)$ & 0.710 & $v\left(4 d \frac{3}{2} \frac{3}{2}\right) \rightarrow \pi\left(4 g \frac{3}{2} \frac{1}{2}\right)$ & 0.392 & $v\left(4 g \frac{9}{2} \frac{7}{2}\right) \rightarrow \pi\left(4 g \frac{9}{2} \frac{5}{2}\right)$ & 23.661 \\
\hline & $\nu\left(4 d \frac{3}{2} \frac{3}{2}\right) \rightarrow \pi\left(4 g \frac{5}{2} \frac{5}{2}\right)$ & 0.321 & $v\left(4 d \frac{5}{2} \frac{1}{2}\right) \rightarrow \pi\left(4 d \frac{3}{2} \frac{1}{2}\right)$ & 0.709 & & \\
\hline
\end{tabular}

Eq. (3.2) are, without exception, larger by factors ranging from $2\left({ }^{100} \mathrm{Mo}\right)$ to $31\left({ }^{96} \mathrm{Zr}\right)$. Note that when projecting out the gauge symmetry, the results for Te isotopes are close to those given here for low values of $g_{p p}$. Comparing the results corresponding to $\chi$ and $g_{p p}$ fixed by fitting either the GT resonance centroid energy or the $\log f t$ value for the $\beta^{+} / E C$ transition of the odd-odd nucleus, and the $\log f t$ value of the $\beta^{-}$decay ending with the $\beta \beta$ daughter nucleus, with those obtained with a renormalized pnQRPA equation and an adjusted Woods-Saxon single-particle mean field, we note that they are close to each other.

Note that for ${ }^{48} \mathrm{Ca}$ the sets of $\left(\chi, g_{p p}\right)$ listed in rows 2 and 3, Table IV, provide half-lives larger than experimental data, which suggests that the value of $\chi$ must be smaller than required by Eq. (3.2). Indeed, decreasing $\chi$ to the value given in the first row of Table IV $(=1.80)$ improves the agreement between the calculated GT resonance energy and the measured GT centroids. Moreover, the agreement with experimental data concerning $T_{1 / 2}$ is also improved. By comparison one can see that the agreement quality obtained in the present paper is similar to that yielded by a full shell-model calculation in Ref. [37]. For this set of dipole interaction strengths, the quenched total strengths of $\beta^{-}$transition of ${ }^{48} \mathrm{Ca}$ and $\beta^{+}$ transition of ${ }^{48} \mathrm{Ti}$ are equal to 15.65 and 2.59 , respectively. The dominant peaks in the $\beta^{-}$distribution correspond to the pnQRPA energies of 6.63 and $12.61 \mathrm{MeV}$. The carried strengths are 1.154 and 3.344, respectively.

An interesting feature for the decay of ${ }^{48} \mathrm{Ca}$ was pointed out by the shell-model studies $[37,54,55]$. This refers to the cumulative effect of the low lying states in ${ }^{48} \mathrm{Sc}$, which actually yield the bulk contribution to the matrix element. The higher $1^{+}$states have a coherent destructive effect on the matrix 
TABLE VII. The same as in Table VI but for the right panels of Figs. 1-4.

\begin{tabular}{|c|c|c|c|c|c|c|}
\hline \multirow[t]{2}{*}{ Nucleus } & \multicolumn{2}{|l|}{ 1st peak } & \multicolumn{2}{|l|}{ 2nd peak } & \multicolumn{2}{|l|}{ 3rd peak } \\
\hline & Transition & Strength & Transition & Strength & Transition & Strength \\
\hline${ }^{48} \mathrm{Ti}$ & $\pi\left(3 p \frac{1}{2} \frac{1}{2}\right) \rightarrow v\left(3 p \frac{3}{2} \frac{3}{2}\right)$ & 0.153 & $\pi\left(3 f \frac{7}{2} \frac{7}{2}\right) \rightarrow v\left(3 f \frac{5}{2} \frac{5}{2}\right)$ & 0.307 & $\pi\left(3 f \frac{7}{2} \frac{3}{2}\right) \rightarrow v\left(3 f \frac{5}{2} \frac{1}{2}\right)$ & 0.562 \\
\hline${ }^{96} \mathrm{Mo}$ & $\pi\left(4 d \frac{5}{2} \frac{1}{2}\right) \rightarrow v\left(4 d \frac{3}{2} \frac{1}{2}\right)$ & 0.146 & $\pi\left(4 d \frac{5}{2} \frac{3}{2}\right) \rightarrow v\left(4 d \frac{5}{2} \frac{5}{2}\right)$ & 0.025 & & \\
\hline${ }^{100} \mathrm{Ru}$ & $\pi\left(4 d \frac{5}{2} \frac{5}{2}\right) \rightarrow v\left(4 g \frac{3}{2} \frac{3}{2}\right)$ & 0.405 & $\begin{array}{l}\pi\left(4 g \frac{9}{2} \frac{9}{2}\right) \rightarrow v\left(4 g \frac{7}{2} \frac{7}{2}\right) \\
\pi\left(4 g \frac{9}{2} \frac{7}{2}\right) \rightarrow v\left(4 g \frac{7}{2} \frac{5}{2}\right)\end{array}$ & $\begin{array}{l}0.509 \\
0.362\end{array}$ & & \\
\hline${ }^{104} \mathrm{Pd}$ & $\pi\left(4 g \frac{9}{2} \frac{5}{2}\right) \rightarrow v\left(4 g \frac{7}{2} \frac{7}{2}\right)$ & 0.441 & $\begin{array}{l}\pi\left(4 g \frac{9}{2} \frac{1}{2}\right) \rightarrow v\left(4 g \frac{9}{2} \frac{3}{2}\right) \\
\pi\left(4 d \frac{5}{2} \frac{1}{2}\right) \rightarrow v\left(4 d \frac{5}{2} \frac{3}{2}\right)\end{array}$ & $\begin{array}{l}0.247 \\
0.110\end{array}$ & $\pi\left(4 g \frac{7}{2} \frac{5}{2}\right) \rightarrow v\left(4 g \frac{9}{2} \frac{7}{2}\right)$ & 0.023 \\
\hline${ }^{110} \mathrm{Cd}$ & $\pi\left(4 d \frac{3}{2} \frac{3}{2}\right) \rightarrow v\left(4 d \frac{5}{2} \frac{1}{2}\right)$ & 0.273 & $\begin{array}{l}\pi\left(4 g \frac{9}{2} \frac{7}{2}\right) \rightarrow v\left(4 g \frac{7}{2} \frac{5}{2}\right) \\
\pi\left(4 d \frac{5}{2} \frac{3}{2}\right) \rightarrow v\left(4 d \frac{3}{2} \frac{3}{2}\right)\end{array}$ & $\begin{array}{l}0.110 \\
0.325\end{array}$ & $\pi\left(4 g \frac{7}{2} \frac{5}{2}\right) \rightarrow v\left(4 g \frac{9}{2} \frac{3}{2}\right)$ & 0.027 \\
\hline${ }^{116} \mathrm{Sn}$ & $\pi\left(4 g \frac{7}{2} \frac{7}{2}\right) \rightarrow v\left(4 g \frac{7}{2} \frac{5}{2}\right)$ & 0.391 & $\begin{array}{l}\pi\left(5 f \frac{7}{2} \frac{7}{2}\right) \rightarrow v\left(5 f \frac{5}{2} \frac{5}{2}\right) \\
\pi\left(4 g \frac{7}{2} \frac{7}{2}\right) \rightarrow v\left(4 g \frac{9}{2} \frac{5}{2}\right)\end{array}$ & $\begin{array}{l}0.117 \\
0.927\end{array}$ & $\pi\left(4 d \frac{5}{2} \frac{1}{2}\right) \rightarrow v\left(4 d \frac{5}{2} \frac{3}{2}\right)$ & 0.019 \\
\hline${ }^{128} \mathrm{Xe}$ & $\begin{aligned} \pi\left(4 d \frac{5}{2} \frac{1}{2}\right) & \rightarrow v\left(4 d \frac{3}{2} \frac{3}{2}\right) \\
\pi\left(5 h \frac{11}{2} \frac{1}{2}\right) & \rightarrow v\left(5 h \frac{11}{2} \frac{3}{2}\right)\end{aligned}$ & $\begin{array}{l}0.011 \\
0.011\end{array}$ & $\begin{array}{l}\pi\left(4 g \frac{9}{2} \frac{5}{2}\right) \rightarrow v\left(4 g \frac{7}{2} \frac{5}{2}\right) \\
\pi\left(4 g \frac{9}{2} \frac{7}{2}\right) \rightarrow v\left(4 g \frac{7}{2} \frac{7}{2}\right)\end{array}$ & $\begin{array}{l}0.020 \\
0.041\end{array}$ & & \\
\hline${ }^{130} \mathrm{Xe}$ & $\begin{aligned} \pi\left(4 d \frac{5}{2} \frac{3}{2}\right) & \rightarrow v\left(4 d \frac{3}{2} \frac{3}{2}\right) \\
\pi\left(5 h \frac{11}{2} \frac{1}{2}\right) & \rightarrow v\left(5 h \frac{11}{2} \frac{3}{2}\right)\end{aligned}$ & $\begin{array}{l}0.020 \\
0.028\end{array}$ & $\pi\left(4 g \frac{9}{2} \frac{7}{2}\right) \rightarrow v\left(4 g \frac{7}{2} \frac{7}{2}\right)$ & 0.026 & & \\
\hline${ }^{134} \mathrm{Ba}$ & $\begin{aligned} \pi\left(5 f \frac{7}{2} \frac{7}{2}\right) & \rightarrow v\left(5 f \frac{5}{2} \frac{5}{2}\right) \\
\pi\left(4 g \frac{9}{2} \frac{3}{2}\right) & \rightarrow v\left(4 g \frac{9}{2} \frac{1}{2}\right)\end{aligned}$ & $\begin{array}{l}0.106 \\
0.086\end{array}$ & & & & \\
\hline${ }^{136} \mathrm{Ba}$ & $\begin{array}{l}\pi\left(4 g \frac{9}{2} \frac{5}{2}\right) \rightarrow v\left(4 g \frac{7}{2} \frac{3}{2}\right) \\
\pi\left(4 g \frac{9}{2} \frac{3}{2}\right) \rightarrow v\left(4 g \frac{7}{2} \frac{1}{2}\right)\end{array}$ & $\begin{array}{l}0.120 \\
0.124\end{array}$ & & & & \\
\hline
\end{tabular}

element. It is worth investigating these aspects within the present formalism. Indeed, in Fig. 5 we plotted the transition amplitude $M_{\mathrm{GT}}$ as a function of the upper limit of energies included in the defining equation (2.21). In other words, for a given $E$ the energy $E_{k}$ defined by Eq. (2.22) and involved in the $M_{\mathrm{GT}}$ expression is restricted by $E_{k} \leqslant E$. We note that in five energy intervals this function is a monotonically increasing function of $E$, while in the following interval the transition amplitude decreases when states of higher energy are added. Also one notices a saturation effect, namely, the contribution of states with energy larger than $16 \mathrm{MeV}$ is very small. One may conclude that our results concerning the behavior of the double $\beta$ transition amplitude are on a par with those of the shell-model calculations. It is remarkable that the maxima of $M_{\mathrm{GT}}(E)$ and $\beta^{-}$strength are reached for similar energies.

Before closing this section we would like to say a few more words about the procedure adopted for fixing the dipole proton-neutron strength parameters. For the sake of a unitary treatment, the half-lives of all double $\beta$ decaying nuclei were calculated by taking for $g_{A}$ the value 1.254. However, in five of the situations considered, the single- $\beta$ properties for the intermediate odd-odd nuclei were determined by supposing an effective value $\left(g_{A}=1\right)$ for the axial-vector coupling strength, which might simulate the contribution of the higher energy states. Thus, although the nuclear matrix elements as well as the proton-neutron interaction strengths are similar for double and single transitions, we considered that the two sets of properties mentioned above are influenced by different parts of the proton-neutron QRPA excitation spectrum. In this context it is worth mentioning that in 1984 Abad et al. [61] advanced the single-state dominance hypothesis (SSDH) which asserts that for double $\beta$ decay processes where the intermediate odd-odd nucleus has the state $1^{+}$as ground state, most of the contribution to the double $\beta$ matrix element comes from the the first intermediate dipole state. If that hypothesis works, then the double $\beta$ process is dominated by two virtual and successive single $\beta^{-}$transitions, one from the ground state of the parent nucleus to the ground state of the intermediate odd-odd nucleus, and the other from there to the ground state of the daughter nucleus. In the meantime the SSDH has been considered by many authors [27,62-68]. As shown in Table X, four odd-odd isotopes have indeed the first $1^{+}$as ground state. Moreover, there are also data available for $\beta^{-}$and $\beta^{+} / E C$ transitions of ${ }^{130} \mathrm{I}$. Therefore, we checked the SSDH validity for all five isotopes from Table II by keeping in Eq. (2.21) only the first term from the sum and considering the states overlap equal to unity. Also the $t_{1 / 2}$ values were calculated by considering $g_{A}=1$. The results are compared with those obtained by summing up over all pnQRPA states, otherwise keeping $g_{A}=1$ in order to have a fair comparison. Results are listed in Table XI. From them one may conclude that our calculations confirm the SSDH for ${ }^{100} \mathrm{Mo},{ }^{104} \mathrm{Ru}$, and ${ }^{110} \mathrm{Pd}$, 
TABLE VIII. The energies of the pnQRPA states which give the largest strength contributions to the peaks in Figs. 1-4, left panels. The carried strengths are also given.

\begin{tabular}{|c|c|c|c|c|c|c|}
\hline \multirow[t]{2}{*}{ Nucleus } & \multicolumn{2}{|c|}{ 1st peak } & \multicolumn{2}{|c|}{ 2nd peak } & \multicolumn{2}{|c|}{ 3rd peak } \\
\hline & pnQRPA energy & Strength & pnQRPA energy & Strength & pnQRPA energy & Strength \\
\hline${ }^{48} \mathrm{Ca}$ & 6.633 & 1.155 & 7.959 & 0.519 & $\begin{array}{l}10.326 \\
12.611\end{array}$ & $\begin{array}{l}0.700 \\
3.344\end{array}$ \\
\hline${ }^{96} \mathrm{Zr}$ & $\begin{array}{l}7.077 \\
7.367\end{array}$ & $\begin{array}{l}0.602 \\
0.467\end{array}$ & $\begin{array}{l}10.316 \\
11.633 \\
11.901\end{array}$ & $\begin{array}{l}1.040 \\
2.473 \\
0.786\end{array}$ & $\begin{array}{l}12.416 \\
13.125\end{array}$ & $\begin{array}{l}5.842 \\
2.212\end{array}$ \\
\hline${ }^{100} \mathrm{Mo}$ & $\begin{array}{l}5.625 \\
5.790 \\
6.452\end{array}$ & $\begin{array}{l}0.428 \\
4.950 \\
1.005\end{array}$ & 9.282 & 1.456 & $\begin{array}{l}11.678 \\
12.104\end{array}$ & $\begin{array}{l}0.702 \\
0.716\end{array}$ \\
\hline${ }^{104} \mathrm{Ru}$ & $\begin{array}{l}5.181 \\
5.444 \\
6.327\end{array}$ & $\begin{array}{l}1.342 \\
2.722 \\
1.321\end{array}$ & $\begin{array}{l}9.028 \\
9.224\end{array}$ & $\begin{array}{l}0.885 \\
0.400\end{array}$ & $\begin{array}{l}11.296 \\
11.670 \\
11.900\end{array}$ & $\begin{array}{l}0.563 \\
1.703 \\
0.266\end{array}$ \\
\hline${ }^{110} \mathrm{Pd}$ & $\begin{array}{l}3.470 \\
5.053 \\
6.339\end{array}$ & $\begin{array}{l}2.554 \\
1.080 \\
2.705\end{array}$ & $\begin{array}{l}10.319 \\
11.067\end{array}$ & $\begin{array}{l}1.028 \\
1.171\end{array}$ & $\begin{array}{l}12.641 \\
12.792\end{array}$ & $\begin{array}{l}1.570 \\
0.294\end{array}$ \\
\hline${ }^{116} \mathrm{Cd}$ & $\begin{array}{l}2.359 \\
3.221\end{array}$ & $\begin{array}{l}1.668 \\
0.462\end{array}$ & $\begin{array}{l}6.042 \\
6.378 \\
7.083\end{array}$ & $\begin{array}{l}0.769 \\
0.927 \\
1.247\end{array}$ & $\begin{array}{l}13.236 \\
13.346 \\
13.407\end{array}$ & $\begin{array}{l}1.121 \\
2.647 \\
1.971\end{array}$ \\
\hline${ }^{128} \mathrm{Te}$ & $\begin{array}{l}5.339 \\
6.880\end{array}$ & $\begin{array}{l}0.445 \\
0.380\end{array}$ & $\begin{array}{l}10.173 \\
10.401\end{array}$ & $\begin{array}{l}0.467 \\
0.198\end{array}$ & $\begin{array}{l}13.713 \\
14.048\end{array}$ & $\begin{array}{r}12.483 \\
7.316\end{array}$ \\
\hline${ }^{130} \mathrm{Te}$ & $\begin{array}{l}6.553 \\
7.965\end{array}$ & $\begin{array}{l}0.462 \\
0.342\end{array}$ & $\begin{array}{l}10.129 \\
10.351\end{array}$ & $\begin{array}{l}0.430 \\
0.291\end{array}$ & $\begin{array}{l}13.652 \\
14.107\end{array}$ & $\begin{array}{r}2.543 \\
19.205\end{array}$ \\
\hline${ }^{134} \mathrm{Xe}$ & 4.188 & 0.713 & $\begin{array}{l}7.403 \\
7.761\end{array}$ & $\begin{array}{l}0.659 \\
0.625\end{array}$ & $\begin{array}{l}12.217 \\
14.865\end{array}$ & $\begin{array}{r}0.784 \\
21.050\end{array}$ \\
\hline${ }^{136} \mathrm{Xe}$ & $\begin{array}{l}7.545 \\
7.902\end{array}$ & $\begin{array}{l}0.710 \\
0.321\end{array}$ & $\begin{array}{l}11.437 \\
12.424\end{array}$ & $\begin{array}{l}0.392 \\
0.709\end{array}$ & 15.359 & 23.661 \\
\hline
\end{tabular}

TABLE IX. The same as in Table VIII, but for the right panels of Figs. 1-4.

\begin{tabular}{|c|c|c|c|c|c|c|}
\hline \multirow[t]{2}{*}{ Nucleus } & \multicolumn{2}{|c|}{1 st peak } & \multicolumn{2}{|c|}{ 2nd peak } & \multicolumn{2}{|c|}{ 3rd peak } \\
\hline & pnQRPA energy & Strength & pnQRPA energy & Strength & pnQRPA energy & Strength \\
\hline${ }^{48} \mathrm{Ti}$ & 5.957 & 0.153 & 6.940 & 0.307 & 7.565 & 0.562 \\
\hline${ }^{96} \mathrm{Mo}$ & 3.361 & 0.146 & 4.224 & 0.025 & & \\
\hline${ }^{100} \mathrm{Ru}$ & 2.099 & 0.405 & $\begin{array}{l}3.617 \\
4.437\end{array}$ & $\begin{array}{l}0.509 \\
0.362\end{array}$ & & \\
\hline${ }^{104} \mathrm{Pd}$ & 0.863 & 0.441 & $\begin{array}{l}4.265 \\
5.300\end{array}$ & $\begin{array}{l}0.247 \\
0.110\end{array}$ & 10.725 & 0.023 \\
\hline${ }^{110} \mathrm{Cd}$ & 1.817 & 0.273 & $\begin{array}{l}4.405 \\
4.531\end{array}$ & $\begin{array}{l}0.110 \\
0.325\end{array}$ & 6.873 & 0.027 \\
\hline${ }^{116} \mathrm{Sn}$ & 1.727 & 0.391 & $\begin{array}{l}5.718 \\
6.378\end{array}$ & $\begin{array}{l}0.117 \\
0.927\end{array}$ & 8.461 & 0.019 \\
\hline${ }^{128} \mathrm{Xe}$ & $\begin{array}{l}2.554 \\
3.246\end{array}$ & $\begin{array}{l}0.011 \\
0.011\end{array}$ & $\begin{array}{l}5.025 \\
5.479\end{array}$ & $\begin{array}{l}0.041 \\
0.020\end{array}$ & & \\
\hline${ }^{130} \mathrm{Xe}$ & $\begin{array}{l}3.356 \\
3.446\end{array}$ & $\begin{array}{l}0.020 \\
0.028\end{array}$ & 4.674 & 0.026 & & \\
\hline${ }^{134} \mathrm{Ba}$ & $\begin{array}{l}3.756 \\
3.803\end{array}$ & $\begin{array}{l}0.106 \\
0.086\end{array}$ & & & & \\
\hline${ }^{136} \mathrm{Ba}$ & $\begin{array}{l}3.242 \\
3.534\end{array}$ & $\begin{array}{l}0.120 \\
0.124\end{array}$ & & & & \\
\hline
\end{tabular}


TABLE X. Experimental energies in keV for the first $1^{+}$states in the intermediate odd-odd nuclei, from Refs. [28-30,32,34, 56-60]. The states in ${ }^{48} \mathrm{Sc}$ and ${ }^{96} \mathrm{Nb}$ at 338 and $1116 \mathrm{keV}$, respectively, are not assigned with angular momentum and parity. Here we, $a d$ hoc, suppose that they have the angular momentum equal to 1 and a positive parity. For ${ }^{136} \mathrm{Cs}$, there are no available data for energy levels. For this case we adopted the same excitation energy for the state $1^{+}$as in ${ }^{134} \mathrm{Cs}$. Also for ${ }^{128,130} \mathrm{I}$, the energies for $1^{+}$ are the same as in Ref. [26].

\begin{tabular}{lcccccccccc}
\hline \hline Nucleus & ${ }^{48} \mathrm{Sc}$ & ${ }^{96} \mathrm{Nb}$ & ${ }^{100} \mathrm{Tc}$ & ${ }^{104} \mathrm{Rh}$ & ${ }^{110} \mathrm{Ag}$ & ${ }^{116} \mathrm{In}$ & ${ }^{128} \mathrm{I}$ & ${ }^{130} \mathrm{I}$ & ${ }^{134} \mathrm{Cs}$ & ${ }^{136} \mathrm{Cs}$ \\
\hline $\mathrm{E}_{1+}(\mathrm{keV})$ & 338 & 1116 & 0 & 0 & 0 & 0 & 58 & 85 & 177 & 177 \\
\hline \hline
\end{tabular}

but not for the remaining two double $\beta$ nuclei, ${ }^{116} \mathrm{Cd}$ and ${ }^{128} \mathrm{Te}$. Most likely for these cases the summation in the expression of $M_{\mathrm{GT}}$ should be extended from one to few states.

We thought it worthwhile to address the question of how stable these results are when the dimension of the singleparticle basis is changed. We checked this feature and obtained a positive result. To be more specific, let us describe the modifications made for ${ }^{110} \mathrm{Pd}$. For this isotope we increased the dimension $D_{1}$ from 23 to 27 and kept the same parameters for single-particle states as before. We changed the pairing strengths in order to preserve the pairing properties, i.e., to have the gap parameters unchanged. The new $\left(G_{p}, G_{n}\right)$ for parent and daughter are $(0.281,0.271)$ and $(0.2795$, $0.1665) \mathrm{MeV}$, respectively. The pnQRPA matrix has the dimension $D_{2}=186$. The ISR value [71] deviates from $N-Z$ by $3 \%$. The proton-neutron interaction strengths have been changed to $\left(\chi, g_{p p}\right)=(0.13735,2.4) \mathrm{MeV}$ in order to keep the $\log f t$ values for the single- $\beta$ transitions of ${ }^{110} \mathrm{Ag}$ close to the experimental data. The results for these observables are 4.84 for $\beta^{-}$and 3.70 for $\beta^{+} / E C$. The double $\beta$ transition amplitude and the $T_{1 / 2}$ obtained under the new circumstances

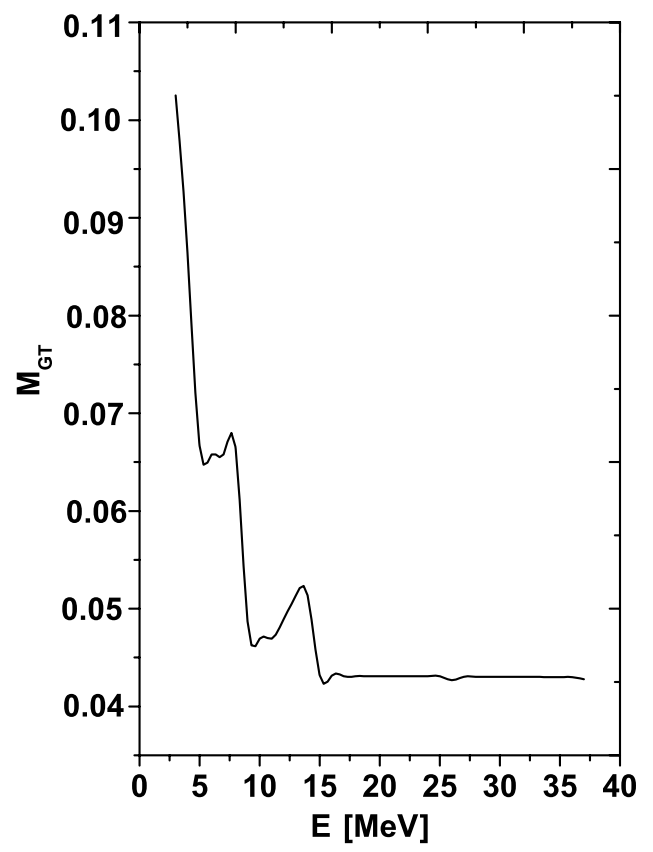

FIG. 5. Double $\beta$ transition amplitude $M_{\mathrm{GT}}$, given by Eq. (2.21), is represented as function of energy $E$ for ${ }^{48} \mathrm{Ca}$. The summation over $k$, in Eq. (2.21), is restricted by $E_{k} \leqslant E$, where $E_{k}$ is defined by Eq. (2.22). are 0.2626 and $15.881 \times 10^{19} \mathrm{yr}$. Note that these values are very close to those listed in Table IV. We conclude that the results are stable against enlarging the single-particle space; moreover, our choice for $D_{1}$ is motivated by the fact that ISR is satisfied.

\section{CONCLUSIONS}

In the previous sections we complete the project started in Ref. [15] by studying the $2 \nu \beta \beta$ decay of another 10 even-even nuclei exhibiting various shapes. In the chosen cases the parent and daughter nuclei have the following shapes: (a) both are spherical, (b) both are deformed prolate, (c) both are deformed oblate, (d) one is spherical and another deformed prolate, (e) both are near spherical but prolate, and (f) both are near spherical but oblate. The deformations obtained for the 10 isotopes are similar to those of Refs. $[49,69]$. In some cases these are different from nuclear deformations reported in Ref. [70]. For example, in the present paper as well as in Ref. [69], the quadrupole deformation for ${ }^{100}$ Mo is negative, while in [70] this is positive. Moreover, as shown in [70] a negative deformation is reached in ${ }^{106} \mathrm{Mo}$. The reason for this discrepancy might be that in [70] the stationary points of the energy function are obtained in the space of quadrupole and hexadecapole deformations while here only the quadrupole variable is considered. Moreover, an angular-momentum-projected single-particle basis is used in this study.

It is evident that an oblate-to-oblate single- $\beta$ transition involves single-particle configurations different from those appearing in a prolate-to-prolate transition. Indeed, suppose that a certain number of nucleons are distributed alternatively

TABLE XI. The $M_{\mathrm{GT}}$ and $t_{1 / 2}$ values obtained with the singlestate dominance hypothesis SSDH. For easy comparison we also give the values obtained within the present formalism. In contrast to the $t_{1 / 2}$ values given in Table IV, the half-lives given here correspond to $g_{A}=1$.

\begin{tabular}{lccccr}
\hline \hline & \multicolumn{2}{c}{ SSDH } & & \multicolumn{2}{c}{ Present } \\
\cline { 2 - 3 } \cline { 5 - 6 } Nucleus & $M_{\mathrm{GT}}$ & $t_{1 / 2}$ & & $M_{\mathrm{GT}}$ & \multicolumn{1}{c}{$t_{1 / 2}$} \\
\hline${ }^{100} \mathrm{Mo}$ & 0.211 & $5.860 \times 10^{19}$ & & 0.305 & $2.82 \times 10^{19}$ \\
${ }^{104} \mathrm{Ru}$ & 0.616 & $7.493 \times 10^{21}$ & & 0.781 & $4.655 \times 10^{21}$ \\
${ }^{110} \mathrm{Pd}$ & 0.551 & $2.208 \times 10^{20}$ & & 0.263 & $9.694 \times 10^{20}$ \\
${ }^{116} \mathrm{Cd}$ & 0.421 & $1.780 \times 10^{19}$ & & 0.116 & $23.63 \times 10^{19}$ \\
${ }^{128} \mathrm{Te}$ & 0.032 & $26.950 \times 10^{24}$ & & 0.090 & $3.38 \times 10^{24}$ \\
\hline \hline
\end{tabular}


in a prolate and an oblate single-particle level and that in the first case the Fermi level for neutrons is characterized by a small quantum number $I$. In this case the $\beta^{-}$strength for the prolate-to-prolate transition is carried by single-particle dipole transitions between states of low $I$ as well as of large $I$ but originating from the upper shell. In contrast, in the oblate-to-oblate transitions, the privileged transitions are those relating neutron and proton single-particle states with large $I$ and those of small $I$ from the upper shell. Such cases can be easily identified in Tables VI and VII. Because of the feature mentioned above, the strength fragmentation is expected to be more pronounced in the oblate-to-oblate transitions. Actually such a situation is met for ${ }^{100} \mathrm{Mo},{ }^{104} \mathrm{Ru},{ }^{110} \mathrm{Pd}$, and ${ }^{116} \mathrm{Cd}$.

The structure of the peaks seen in Figs. 1 and 2 in the $\beta^{-}$ strength distribution of the nuclei just mentioned is as follows. In ${ }^{100}$ Mo the peaks are determined by transitions inside shells $1 g$ (first peak), $2 d$ (second peak), and $1 g$ and $1 f$ (third peak). For ${ }^{104} \mathrm{Ru}$ the first two peaks are determined mainly by the transitions from shell $1 g$ while the third one by the transition from shell $1 f$. In ${ }^{110} \mathrm{Pd}$ the following shells are involved in the transitions contributing most to the three peaks: $1 g$ (first peak); $1 g, 2 d$ (second peak); and $1 g, 1 f$ (third peak). For ${ }^{116} \mathrm{Cd}$ only one shell contributes most to any of the three dominant peaks: $1 g$ (first and second peaks), $1 f$ (third peak).

Note that for $\mathrm{Te}$ and $\mathrm{Xe}$ isotopes, the $\beta^{-}$strength is mainly concentrated in one pnQRPA state. These nuclei are almost spherical ( $\mathrm{Te}$ isotopes are soft prolate while $\mathrm{Xe}$ isotopes are soft oblate). Moreover, in the daughter nuclei the nuclear deformation has the same sign as in the corresponding parent nuclei. In ${ }^{128} \mathrm{Te}$ and ${ }^{130} \mathrm{Te}$ the dominant single-particle state $n p$ transitions are $v\left(4 g \frac{9}{2} \frac{5}{2}\right) \rightarrow \pi\left(4 g \frac{7}{2} \frac{7}{2}\right)$ and $\nu\left(4 d \frac{5}{2} \frac{3}{2} \rightarrow\right.$ $\pi\left(4 d \frac{3}{2} \frac{3}{2}\right)$, respectively. In Xe isotopes the $n p$ single-particle transition $\nu\left(4 g \frac{9}{2} \frac{7}{2}\right) \rightarrow \pi\left(4 g \frac{9}{2} \frac{5}{2}\right)$ prevails.

The transition amplitudes $M_{\mathrm{GT}}$ and half-lives for the $2 \nu \beta \beta$ process are calculated within the pnQRPA approach by using a projected spherical basis. The agreement with the available data is quite good. The adopted fitting procedure for the $p n$ dipole interaction strengths yield large values for $g_{p p}$, in several cases. These values are not far from the critical value, where the pnQRPA breaks down. It is an open question whether for these transitions a good agreement with the data would be possible by keeping a small $g_{p p}$ but accounting for higher pnQRPA effects. Inspecting Table IV, one can judge not only the agreement of the present results with the experimental data but also the comparison between predictions of different theoretical approaches. Indeed, the agreement with experimental data is reasonably good. Although they are based on different formalisms as well as different single-particle states the present results and those of Suhonen et al. $[1,45,46]$ are not far from each other. Comparing the results of the present paper, obtained with $\chi$ and $g_{p p}$ given by Eq. (3.2), and the corresponding predictions from Refs. $[35,36]$, one notices that they are quite different.

It is worth mentioning that for ${ }^{104} \mathrm{Ru}$ and ${ }^{110} \mathrm{Pd}$ the $p n$ dipole interactions are fully determined by fitting the data concerning the $\beta^{+} / E C$ and $\beta^{-}$decay $\log f t$ values of the ground state $\left(1^{+}\right)$of the intermediate nuclei ${ }^{104} \mathrm{Rh}$ and ${ }^{110} \mathrm{Ag}$, respectively. The predictions for the double $\beta$ emitter half-lives are $0.76 \times 10^{21}$ and $15.85 \times 10^{19} \mathrm{yr}$. They are 40 and 8 times smaller than the corresponding predictions of Ref. [36]. Our prediction for the half-life of ${ }^{134} \mathrm{Xe}$, against double $\beta$ decay, is $3.75 \times 10^{24} \mathrm{yr}$ which exceeds by a factor 15 the corresponding finding in $[35,48]$.

The single-state dominance is confirmed, by our formalism, to be valid for ${ }^{100} \mathrm{Mo},{ }^{104} \mathrm{Ru}$, and ${ }^{110} \mathrm{Pd}$.

Finally, we conclude that the projected spherical singleparticle basis provides a suitable framework for a unified description of the double $\beta$ properties of spherical and deformed nuclei. The results presented in [15] and here constitute a good starting point for studying the higher pnQRPA contributions to the $2 \nu \beta \beta$ process as well as the transitions populating the daughter nuclei in an excited state [72].
[1] J. Suhonen and O. Civitarese, Phys. Rep. 300, 123 (1998).

[2] J. D. Vergados, Phys. Rep. 361, 1 (2001).

[3] H. Primakoff and S. Rosen, Rep. Prog. Phys. 22, 121 (1959).

[4] W. C. Haxton and G. J. Stephenson, Jr., Prog. Part. Nucl. Phys. 12, 409 (1984).

[5] T. Tomoda, Rep. Prog. Phys. 54, 53 (1991).

[6] A. Faessler, Prog. Part. Nucl. Phys. 21, 183 (1988).

[7] H. V. Klapdor-Kleingrothaus, Sixty Years of Double $\beta$ Decay: from Nuclear Physics to beyond Standard Model Particle Physics (World Scientific, Singapore, 2001).

[8] A. A. Raduta, Prog. Part. Nucl. Phys. 48, 233 (2002).

[9] A. A. Raduta, A. Faessler, and D. S. Delion, Nucl. Phys. A564, 185 (1993); Phys. Lett. B312, 13 (1993).

[10] A. A. Raduta, D. S. Delion, and A. Faessler, Nucl. Phys. A617, 176 (1997).

[11] H. Homma, E. Bender, M. Hirsch, K. Muto, H. V. KlapdorKleingrothaus, and T. Oda, Phys. Rev. C 54, 2972 (1996).

[12] P. Sarriguren, E. Moya de Guerra, A. Escuderos, and A. C. Carrizo, Nucl. Phys. A635, 55 (1998); P. Sarriguren,
E. Moya de Guerra, and A. Escuderos, ibid. A658 13 (1999); A691, 631 (2001); Phys. Rev. C 64, 064306 (2001); P. Sarriguren, E. Moya de Guerra, L. Pacearescu, A. Faessler, F. Simkovic, and A. A. Raduta, ibid. 67, 044313 (2003).

[13] J. Engel, M. Bender, J. Dobaczewski, W. Nazariewicz, and R. Surnam, Phys. Rev. C 60, 014302 (1999); M. Bender, J. Dobaczewski, J. Engel, and W. Nazarewicz, ibid. 65, 054322 (2002).

[14] F. Simkovic, L. Pacearescu, and A. Faessler, Nucl. Phys. A733, 321 (2004).

[15] A. A. Raduta, A. Escuderos, A. Faessler, E. Moya de Guerra, and P. Sarriguren, Phys. Rev. C 69, 064321 (2004).

[16] A. A. Raduta, D. S. Delion, and N. Lo Iudice, Nucl. Phys. A564, 185 (1993).

[17] A. A. Raduta, N. Lo Iudice and I. I. Ursu, Nucl. Phys. A584, 84 (1995).

[18] A. A. Raduta, A. H. Raduta, and A. R. Raduta, Phys. Rev. B 59, 8209 (1999); A. A. Raduta, E. Garrido, and E. Moya de Guerra, Eur. Phys. J. D 15, 65 (2001). 
[19] A. A. Raduta, A. Escuderos, and E. Moya de Guerra, Phys. Rev. C 65, 024312 (2002).

[20] S. G. Nilsson, Dan. Mat. Fys. Medd. 29 (16) (1955).

[21] M. E. Rose, Elementary Theory of Angular Momentum (Wiley, New York, 1957).

[22] P. Ring and P. Schuck, The Nuclear Many-Body Problem (Springer-Verlag, Berlin, Heidelberg, New York, 1980), p. 76.

[23] K. Grotz and H. V. Klapdor, Nucl. Phys. A460, 395 (1986).

[24] P. Moller and J. Randrup, Nucl. Phys A514, 1 (1990).

[25] E. Bender, K. Muto, and H. V. Klapdor, Phys. Lett. B208, 53 (1988).

[26] R. Madey, B. S. Flanders, B. D. Anderson, A. R. Baldwin, J. W. Watson, S. M. Austin, C. C. Foster, H. V. Klapdor, and K. Grotz, Phys. Rev. C 40, 540 (1989).

[27] A. Garcia et al., Phys. Rev. C 47, 2910 (1993).

[28] Balraj Singh, Nuclear Data Sheets 81, 1 (1997).

[29] Jean Blachot, Nuclear Data Sheets 64, 1 (1991).

[30] D. De Frenne and E. Jacobs, Nucl. Data Sheets 89, 481 (2000).

[31] M. Bhattacharya, A. Garcia, M. Hindi, E. Norman, C. Ortiz, N. Kaloskamis, C. Davids, O. Civitarese, and J. Suhonen, Phys. Rev. C 58, 1247 (1998).

[32] Jean Blachot, Nucl. Data Sheets 92, 455 (2001).

[33] H. Miyahara et al., Nucl. Instrum. Methods Phys. Res. A 353, 229 (1995); C. M. Lederer and V. S. Shirley, Table of Isotopes, 7th ed. (Wiley, New York, 1978), p. 631.

[34] M. Kanbe and K. Kitao, Nucl. Data Sheets 94, 227 (2001).

[35] K. Grotz and H. V. Klapdor, Phys. Lett. B157, 242 (1985).

[36] M. Hirsch, X. R. Wu, H. V. Klapdor-Kleingrothaus, Ching Cheng-rui, and Ho Tso-hsiu, Phys. Rep. 242, 403 (1994).

[37] L. Zhao, B. A. Brown, and W. A. Richter, Phys. Rev. C 42, 1120 (1990).

[38] H. V. Klapdor, Prog. Part. Nucl. Phys. 17, 419 (1986).

[39] S. R. Elliot and P. Vogel, Ann. Rev. Nucl. Part. Sci. 52, 115 (2002); A. S. Barabash, Czech. J. Phys. 52, 567 (2002).

[40] H. Ejiri et al., Phys. Lett. B258, 17 (1991).

[41] T. Kirsten, E. Heusser, D. Kaether, J. Oehm, E. Pernicka, and H. Richter, in Proceedings of the International Symposium on Nuclear $\beta$ Decays and the Neutrino, Osaka, Japan, June 1986, edited by T. Kotani, H. Ejiri, and E. Takasugi (World Scientific, Singapore, 1986), p. 81.

[42] S. I. Vasil'ev, A. A. Klimenko, S. B. Osetrov, A. A. Pomansky, and K. A. Smol'nikov, JETP Lett. 51, 622 (1990).

[43] E. W. Hennecke, O. K. Manuel, and D. D. Sabu, Phys. Rev. C 11, 1378 (1975).

[44] W. J. Lin et al., Nucl. Phys. A481, 477 (1988).

[45] J. Suhonen and O. Civitarese, Phys. Rev. C 49, 3055 (1994).
[46] M. Aunola and J. Suhonen, Nucl. Phys. A602, 133 (1996).

[47] H. V. Klapdor and K. Grotz, Phys. Lett. B142, 323 (1984); A. Balysh et al., ibid. B356, 450 (1995).

[48] X. R. Wu, M. Hirsch, A. Staudt, H. V. Klapdor-Kleingrothaus, Cheng-Rui Ching, and Tso-Hsui Ho, Commun. Theor. Phys. 20, 453 (1993).

[49] G. Audi and A. H. Wapstra, Nucl. Phys. A595, 409 (1995); G. Audi, O. Bersillon, J. Blachot, and A. H. Wapstra, ibid. A624, 1 (1997).

[50] H. Akimune, H. Ejiri, M. Fujiwara, I. Daito, T. Inomata, R. Hazama, A. Tamii, H. Toyokawa, and M. Yosoi, Phys. Lett. B394, 23 (1997).

[51] B. D. Anderson, T. Chittrakarn, A. R. Baldwin, C. Lebo, R. Madey, P. C. Tandy, J. W. Watson, B. A. Brown, and C. C. Foster, Phys. Rev. C 31, 1161 (1985).

[52] L. Zamick and N. Auerbach, Phys. Rev. C 26, 2185 (1982).

[53] D. Cha, Phys. Rev. C 27, 2269 (1987).

[54] D. Zheng, L. Zamick, and N. Auerbach, Ann. Phys. 197, 343 (1990).

[55] E. Caurier, A. Poves, A. P. Zuker, Phys. Lett. B252, 13 (1990).

[56] T. W. Burrow, Nucl. Data Sheets 68, 1 (1993).

[57] L. K. Pekev, Nucl. Data Sheets 68, 165 (1993).

[58] Balraj Singh, Nucl. Data Sheets 93, 33 (2001).

[59] Yu. V. Sergeenkov, Nucl. Data Sheets 71, 557 (1994).

[60] A. A. Sonzogni, Nucl. Data Sheets 95, 837 (2002).

[61] J. Abad, A. Morales, R. Nunes-Lagos, and A. F. Pacheco, Ann. Fis. A 80, 9 (1984).

[62] H. Ejiri, Nucl. Phys. A577, 399c (1994).

[63] O. Civitarese and J. Suhonen, Phys. Rev. C 58, 1535 (1998).

[64] O. Civitarese and J. Suhonen, Nucl Phys. A653, 321 (1998).

[65] F. Simkovic, P. Domin, and S. V. Semenov, J. Phys. G: Nucl. Part. Phys. 27, 2233 (2001).

[66] H. Ejiri, Prog. Part. Nucl. Phys. 48, 185 (2002).

[67] O. Civitarese, J. Suhonen, and H. Ejiri, Eur. Phys. J. A 16, 353 (2003).

[68] P. Domin, S. Kovalenko, F. Simkovic, and S. V. Semenov, arXiv: nucl-th/0411002 v1.

[69] K. Grotz and H. V. Klapdor, Nucl. Phys. A460, 395 (1986).

[70] G. A. Lalazissis, S. Raman, and P. Ring, At. Data Nucl. Data Tables 60, 177 (1995).

[71] K. Ikeda, Prog. Theor. Phys. 31434 (1964); K. Ikeda, S. Fujita, and J. I. Fujita, Phys. Lett. 3, 271 (1963); J. I. Fujita and K. Ikeda, Prog. Theor. Phys. 36, 288 (1966).

[72] A. A. Raduta, A. Faessler, and S. Stoica, Nucl. Phys. A534, 149 (1991). 